\title{
Under the FIRElight: Stellar Tracers of the Local Dark Matter Velocity Distribution in the Milky Way
}

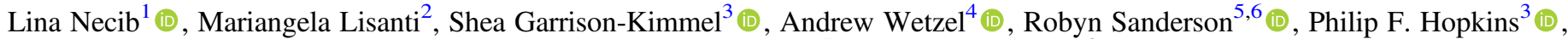 \\ Claude-André Faucher-Giguère ${ }^{7}$ (iD), and Dušan Kereš ${ }^{8}$ (iD \\ ${ }^{1}$ Walter Burke Institute for Theoretical Physics, California Institute of Technology, Pasadena, CA 91125, USA \\ ${ }^{2}$ Department of Physics, Princeton University, Princeton, NJ 08544, USA \\ ${ }^{3}$ TAPIR, California Institute of Technology, Pasadena, CA 91125, USA \\ ${ }^{4}$ Department of Physics, University of California, Davis, CA 95616, USA \\ ${ }^{5}$ Department of Physics and Astronomy, University of Pennsylvania, Philadelphia, PA 19104, USA \\ ${ }^{6}$ Center for Computational Astrophysics, Flatiron Institute, New York, NY 10010, USA
${ }^{7}$ Department of Physics and Astronomy and CIERA, Northwestern University, Evanston, IL 60208, USA \\ ${ }^{8}$ Department of Physics, Center for Astrophysics and Space Sciences, University of California at San Diego, La Jolla, CA 92093, USA \\ Received 2019 February 15; revised 2019 August 9; accepted 2019 August 12; published 2019 September 18
}

\begin{abstract}
The Gaia era opens new possibilities for discovering the remnants of disrupted satellite galaxies in the solar neighborhood. If the population of local accreted stars is correlated with the dark matter sourced by the same mergers, one can then map the dark matter distribution directly. Using two cosmological zoom-in hydrodynamic simulations of Milky-Way-mass galaxies from the Latte suite of the FIRE-2 simulations, we find a strong correlation between the velocity distribution of stars and dark matter at the solar circle that were accreted from luminous satellites. This correspondence holds for dark matter that is either relaxed or in a kinematic substructure called debris flow, and is consistent between two simulated hosts with different merger histories. The correspondence is more problematic for streams because of possible spatial offsets between the dark matter and stars. We demonstrate how to reconstruct the dark matter velocity distribution from the observed properties of the accreted stellar population by properly accounting for the ratio of stars to dark matter contributed by individual mergers. This procedure does not account for the dark matter that originates from nonluminous satellites, which may constitute a nontrivial fraction of the local contribution. After validating this method using the FIRE-2 simulations, we apply it to the Milky Way and use it to recover the dark matter velocity distribution associated with the recently discovered stellar debris field in the solar neighborhood. Based on results from Gaia, we estimate that $42_{-22}^{+26} \%$ of the local dark matter that is accreted from luminous mergers is in debris flow.
\end{abstract}

Key words: dark matter - Galaxy: formation - Galaxy: kinematics and dynamics - stars: kinematics and dynamics

\section{Introduction}

In the $\Lambda$ CDM paradigm, a dark matter (DM) host halo is built up hierarchically from galaxy mergers (White \& Rees 1978; Diemand et al. 2008; Springel et al. 2008; Klypin et al. 2011). These satellites also contribute stars, which may hold clues to the underlying DM distribution in the Milky Way. In this work, we use simulations of Milky-Way-mass galaxies from the FEEDBACK IN REALISTIC ENVIRONMENTS (FIRE) ${ }^{9}$ project (Hopkins et al. 2018) to study the correlation between accreted stars and DM, and its dependence on galactic merger history.

The chemical abundance and phase-space distribution of an accreted stellar population can be used to infer the properties of its parent galaxy (Helmi et al. 2003; Bullock \& Johnston 2005; Robertson et al. 2005; Font et al. 2006; De Lucia \& Helmi 2008; Deason et al. 2016). In this fashion, Belokurov et al. (2018) and Helmi et al. (2018) argued that the population of local accreted stars consists predominantly of debris from a disrupted satellite galaxy with original stellar mass $M_{* \text {,total }} \sim 10^{7}-10^{8} M_{\odot}$. This merger can potentially explain the observed density break in the halo at Galactocentric radii of $\sim 20 \mathrm{kpc}$ (Deason et al. 2018), as well as the population of globular clusters on highly radial orbits (Myeong et al. 2018b). Referred to as the Gaia Sausage or Gaia Enceladus, this

\footnotetext{
9 http://fire.northwestern.edu
}

substructure comprises the majority of the local distribution of accreted stars (identified by both metallicity and kinematics), with the remaining fraction appearing to be nearly isotropic and metal poor.

Necib et al. (2019) showed that these findings have important implications for the local DM distribution, as they suggest that a nontrivial fraction is in the substructure. This argument depends on whether stars that are tidally stripped from a satellite galaxy trace the DM that is removed from the same source. The DM-stellar correspondence is not guaranteed for a variety of reasons. First, stars are typically more tightly bound than DM toward the center of a galaxy, and thus have a different initial phase-space structure. In an extreme case, a cuspy DM halo can admit a cored stellar distribution (Breddels \& Helmi 2013). Additionally, the majority of stars are stripped only after the majority of the DM because the latter is preferentially removed in the initial stages of satellite disruption. Second, the mass-to-light ratio varies by orders of magnitude between galaxies (McConnachie 2012), so the relative mass of stars to DM that each contributes differs. Therefore, even if one satellite contributes a significant fraction of accreted stars, it may not contribute an equivalent fraction of DM. These effects can be further exacerbated when restricting to a spatial volume like the solar neighborhood.

In this work, we demonstrate how to reconstruct the properties of DM that is accreted from luminous satellites. To organize the discussion, we classify the DM into three separate 
components that are delineated by relative accretion time. The first component includes DM that was accreted at redshifts $z \gtrsim 3$ from the oldest mergers. We refer to this component as "relaxed" in this work, though it has also been referred to as "virialized" in the literature. Herzog-Arbeitman et al. (2018a) demonstrated that this old DM population is well traced by metal-poor stars using the ERIS hydrodynamic simulation (Guedes et al. 2011). In this case, convergence in the velocity distributions was reached for stars with iron abundance $[\mathrm{Fe} / \mathrm{H}] \lesssim-3$. This result motivated a first study using the RAVE-TGAS data set to recover the velocity distribution of the local relaxed DM component (Herzog-Arbeitman et al. 2018b).

We divide DM accreted from younger mergers into two separate categories: debris flow and streams. Debris flow is an example of kinematic substructure that is spatially mixed on large scales. It arises from the accretion of one or more older satellites that completed several orbital wraps (Kuhlen et al. 2012; Lisanti \& Spergel 2012). In this case, any structure in position space is washed out, while velocity-space features are preserved (Helmi et al. 1999; Gómez et al. 2010). The properties of debris flow are quite similar between stars and DM, likely because the tidal debris is older and therefore more well mixed (Lisanti et al. 2015). These conclusions are based on studies of the Via Lactea DM-only simulation (Diemand et al. 2008), where star "particles" were painted onto the mostbound DM "particles" in the satellite. It should be repeated using a full hydrodynamic simulation, as we do here.

Streams, in contrast, are relics of the youngest mergers and are neither spatially nor kinematically mixed. They result from tidal debris that is torn off a satellite as it completes a small number of orbits (Diemand et al. 2008; Vogelsberger et al. 2009; Zemp et al. 2009; Kuhlen et al. 2010; Elahi et al. 2011; Maciejewski et al. 2011; Vogelsberger \& White 2011). For these accretion events, the stars may not necessarily act as adequate tracers for the DM as has been noted in simulations of merging dwarf galaxies (Peñarrubia et al. 2008) or of the Sagittarius stream (Purcell et al. 2012).

In this work, we study the correlation between stars and DM accreted from luminous satellites in two Milky-Way-mass halos with differing merger histories. These two simulated galaxies share general properties of the Galactic disk and stellar halo (Sanderson et al. 2018b), and are thus excellent systems in which to study the DM-stellar correlations of interest here. Our approach is to identify the stars and DM that originate from a given satellite galaxy and follow them as a function of time to see where they eventually end up relative to each other. We find that stars from the oldest mergers trace the relaxed DM. Stars and DM in debris flow are also well correlated. The correspondence is not as robust for younger mergers leaving behind streams, because spatial offsets between the DM and stars can lead to localized variations in their velocity components.

We demonstrate how to recover the DM distribution in the solar neighborhood in cases where it is dominated by a relaxed population and debris flow. After successfully demonstrating this procedure with simulations from the FIRE project, we apply it to the Milky Way and the recently discovered debris field in the solar neighborhood. This procedure pertains specifically to DM accreted from luminous satellites and therefore does not account for contributions from nonluminous satellites, which requires further study. The nonluminous satellites can contribute a nontrivial fraction of the local DM. Additionally, the conclusions are specific to the solar circle (defined as $\left|r-r_{\odot}\right|<2 \mathrm{kpc}$ and $|z| \leqslant 1.5 \mathrm{kpc}$ with $r_{\odot}$ the solar radius), which is the volume studied in this work.

This paper is organized as follows. Section 2 introduces the FIRE simulations and provides more details about the two host halos studied in this work. Section 3 describes the breakdown of the DM and stars within the solar circle of the hosts in terms of their accretion time and progenitor characteristics. Section 4 discusses the correlation between the stars and DM for the relaxed, debris flow, and stream categories described above. Section 5 demonstrates how to build the total DM distribution; this new strategy is applied to the Milky Way in Section 6. We conclude in Section 7. The Appendix includes additional figures that supplement the main results of the paper.

\section{FIRE-2 Simulations}

\subsection{The Host Halos}

We analyze two cosmological zoom-in (Katz \& White 1993; Onorbe et al. 2014) hydrodynamic simulations from the Latte suite (Wetzel et al. 2016) of the FIRE-2 simulations (Hopkins et al. 2018). FIRE-2 simulations are run using the GIZMO code $^{10}$ (Hopkins 2015) with the mesh-free finite-mass ("MFM") Lagrangian Godunov method for hydrodynamics, while gravity is solved using a version of the Tree-PM solver from GADGET-3 (Springel 2005). We briefly review the details of these simulations that are most relevant for our study; see Hopkins et al. (2018) and Sanderson et al. (2018b) for more details.

FIRE-2 simulations include heating from a metagalactic background (Faucher-Giguère et al. 2009) and cooling from local stellar sources from $T \sim 10-10^{10} \mathrm{~K}$. Star formation occurs in locally self-gravitating (Hopkins et al. 2013), Jeansunstable, self-shielding (Krumholz \& Gnedin 2011) molecular gas. Stellar feedback occurs through photoionization, photoelectric heating, radiation pressure, supernovae Ia and II, and stellar winds from primarily $\mathrm{O}, \mathrm{B}$, and AGB stars. Inputs are taken directly from stellar evolution models using STARBURST 99 V7. 0 (Leitherer et al. 1999, 2014) and assume the Kroupa (2001) IMF. The Latte simulations that we use also include subgrid turbulent diffusion of metals in gas ( $\mathrm{Su}$ et al. 2017; Hopkins et al. 2018), which produces more realistic metallicity distributions (Escala et al. 2018).

We focus on the galaxies m12i (introduced in Wetzel et al. 2016) and m12f (introduced in Garrison-Kimmel et al. 2017b), which provide contrasting formation histories: the latter experiences more mergers at late cosmic times. Both m12i and m12f assume a $\Lambda$ CDM cosmology with $\Omega_{\Lambda}=0.728$, $\Omega_{m}=0.272, \quad \Omega_{b}=0.0455, \quad h=0.702, \quad \sigma_{8}=0.807, \quad$ and $n_{s}=0.961$. The initial mass of baryonic particles is $7070 M_{\odot}$ (though because of stellar mass loss, the typical star particle has mass $\approx 5000 M_{\odot}$ at redshift $z=0$ ); the gravitational softening length is $4 \mathrm{pc}$ (Plummer equivalent) for stars, and gas has adaptive softening/smoothing down to $1 \mathrm{pc}$. DM particles in the zoom-in region have mass $3.5 \times 10^{4} M_{\odot}$ and softening length of $40 \mathrm{pc}$.

At redshift $z=0$, the primary host halo in $\mathrm{m} 12 \mathrm{i}$ has $M_{200 \mathrm{~m}}=1.2 \times 10^{12} M_{\odot}$ and $R_{200 \mathrm{~m}}=336 \mathrm{kpc}$, defined via the radius containing 200 times the average matter density. Within this radius, the host halo contains $N_{\text {particle }}=5.08 \times 10^{7}$

\footnotetext{
${ }^{10}$ http://www.tapir.caltech.edu/ phopkins/Site/GIZMO.html
} 
DM, gas, and star particles. The corresponding properties for the host halo in $\mathrm{m} 12 \mathrm{f}$ are as follows: $M_{200 \mathrm{~m}}=1$. $7 \times 10^{12} M_{\odot}, R_{200 \mathrm{~m}}=380 \mathrm{kpc}$, and $N_{\text {particle }}=7.44 \times 10^{7}$. Each host halo is selected to be isolated, with no equally massive halos within $5 R_{200 \mathrm{~m}}$.

The host galaxies of $\mathrm{m} 12 \mathrm{i}$ and $\mathrm{m} 12 \mathrm{f}$ are similar in many respects to the Milky Way (Sanderson et al. 2018b). For example, the total stellar mass of the Galactic disk is $(5 \pm 1) \times 10^{10} M_{\odot}$ (Bland-Hawthorn \& Gerhard 2016), compared to $5.5 \times 10^{10}$ and $6.9 \times 10^{10} M_{\odot}$ in $\mathrm{m} 12 i$ and $\mathrm{m} 12 \mathrm{f}$, respectively (this differs from the total mass inside $R_{200 \mathrm{~m}}$ as it excludes satellites). Additionally, these simulations provide a reasonable match to the observed morphology of Milky-Waylike galaxies (Garrison-Kimmel et al. 2018; Sanderson et al. 2018b), disk kinematics and abundance gradients (Ma et al. 2017), satellite dwarf galaxy stellar masses, velocity dispersions, metallicities, and star formation histories (Wetzel et al. 2016; Escala et al. 2018; Garrison-Kimmel et al. 2019), and properties of the thick disk and stellar halo (Bonaca et al. 2017; Sanderson et al. 2018a).

We identify DM (sub)halos using the ROCKSTAR phasespace finder ${ }^{11}$ (Behroozi et al. 2013b), and we generate merger trees using CONSISTENTTREES (REF) across 600 snapshots from redshifts $z=0-99$. We ran the halo finder on only the DM particles, and we assigned stars to each halo in postprocessing (see below).

\subsection{Tracking Dark Matter and Stars}

To understand the origin of stars and DM near the solar circle, we track the location of DM/star particles over all snapshots. To start, we identify all of the DM particles in the solar circle of the host $\left(\left|r-r_{\odot}\right|<2 \mathrm{kpc}\right.$ and $\left.|z| \leqslant 1.5 \mathrm{kpc}\right)$ at the present day. We then follow the location of every particle at each previous snapshot, checking if it falls within the virial radius $R_{200 \mathrm{~m}}$ of a (sub)halo and if its velocity lies within $3 \sigma$ of the (sub)halo's internal velocity (i.e., the maximum between its maximum circular velocity and its velocity dispersion). If these conditions are met, we mark the (sub)halo as the particle's host, further requiring that the DM is associated with the same (sub) halo for three out of the last four snapshots to avoid contamination by flybys that happen to fall within the velocity dispersion. We mark $z_{\text {acc }}$ as the last redshift at which the particle was bound to the (sub)halo; the particle is bound to the primary host halo in the following snapshot. These requirements lead to an unassociated DM fraction of $47 \%$ (59\%) for m12i (m12f), which we define as DM particles that have never been associated with a halo.

The procedure to associate stars to each subhalo is similar. A star particle must lie within a subhalo's virial radius ${ }^{12}$ and have a velocity that falls within $2.5 \sigma$ of the subhalo's stellar velocity dispersion (computing membership and velocity dispersion iteratively until convergence). We include as "galaxies" only subhalos that contain at least 10 stars. We also require that a star particle is part of the same subhalo for at least three out of the last four snapshots. We quote the stellar mass of a given subhalo at the particle's $z_{\text {acc}}$.

\footnotetext{
11 https://bitbucket.org/pbehroozi/rockstar-galaxies

12 We make sure the star particles are within the subhalo's virial radius instead of the inner part of the subhalo as the subhalo's virial radius changes during disruption, making this a safer criteria for associating stars across time. A maximum distance cut of $30 \mathrm{kpc}$ is also required.
}

In this manner, we identify the subhalo progenitor of each $\mathrm{DM} /$ star particle observed today in the solar circle of the primary host galaxy. We also store information on the progenitor subhalo, such as its total DM and stellar mass. Because of tidal stripping, the total mass of a subhalo at $z_{\text {acc }}$ is typically smaller than its initial mass before falling into the primary host. Thus, we also use the subhalo peak mass, $M_{\text {peak }}$, computed from the merger trees.

There are two important resolution effects that affect our ability to track all of the DM and star particles in the solar circle. First, there is a minimum mass for luminous subhalos in the simulation set by the mass of each star particle $\left(\sim 5000 M_{\odot}\right.$ at redshift $z=0$ ). Because we only track galaxies with at least 10 star particles, this leads to the effective lower limit on the total stellar mass of a satellite to be $\sim 10^{5} M_{\odot}$, which corresponds to a halo mass of $\sim 5 \times 10^{8} M_{\odot}$. Thus, we conservatively label the subset of subhalos with $M_{\text {peak }} \gtrsim$ $10^{9} M_{\odot}$ to be luminous in this work.

Second, there is a minimum (sub)halo mass of $\sim 10^{6} M_{\odot}$ because of the DM mass resolution. When tracking the origin of a DM particle, we may find that it is not associated with a specific progenitor. This may either be because its (sub)halo is not resolved or because the DM was never associated with a (sub)halo and was accreted smoothly. We cannot distinguish between these two possibilities.

Throughout the paper, we will separate the DM into two components. The first is the component that originates from luminous subhalos (i.e., with at least 10 stars) with $M_{\text {peak }}>10^{9} M_{\odot}$. The second is the component that originates from either a subhalo whose galaxy was not adequately resolved, a subhalo too small to have formed stars (dark subhalo), an unresolved subhalo, or smooth accretion. We will refer to this component as "Dark/Unresolved."

\section{Accretion History at the Solar Circle}

Because the primary focus of this work is the local DM velocity distribution, we restrict the study of $\mathrm{m} 12 i$ and m12f to the volume within distances $|z| \leqslant 1.5 \mathrm{kpc}$ of the midplane and galactocentric radii $r_{\odot} \pm 2 \mathrm{kpc}$, where $r_{\odot}=$ $8 \mathrm{kpc}$. This is justified because the scale radii of the simulated disks are comparable to those of the Milky Way (Sanderson et al. 2018a). We refer to this volume as the "solar circle." A total of $\sim 1.70 \times 10^{5}\left(2.19 \times 10^{5}\right) \mathrm{DM}$ and $\sim 9.78 \times 10^{5}\left(1.48 \times 10^{6}\right)$ star particles are within this region of $\mathrm{m} 12 \mathrm{i}$ (m12 $\mathrm{f})$.

The total fraction of accreted stars at redshift $z=0$ constitutes only $1.5 \%(2.6 \%)$ of all stars in the solar circle of m12i (m12 f). ${ }^{13}$ The vast majority of the stars are born in situ-that is, they are born within the host galaxy (Zolotov et al. 2009; Font et al. 2011; McCarthy et al. 2012; Cooper et al. 2015; Pillepich et al. 2015; Bonaca et al. 2017). However, the fraction of accreted stars increases toward lower metallicities. The probability of a star being accreted with a metallicity $[\mathrm{Fe} / \mathrm{H}]<-2$ is $69 \%$ $(89 \%)$ for $\operatorname{m12} i(\mathrm{~m} 12 \mathrm{f})$. This increases to $96 \%$ (96\%) for m12i (m12f) when requiring $[\mathrm{Fe} / \mathrm{H}]<-3$.

Table 1 lists the top four satellite galaxies that contribute the greatest fraction of accreted stellar mass at the solar circle of m12i. We see that $40 \%$ of these stars were accreted between redshifts of $z_{\text {acc }}=2.04-2.34$ from a $3.6 \times 10^{10} M_{\odot}$ satellite.

\footnotetext{
13 Note that when we refer to "accreted stars," we do not include stars that formed from accreted gas, but rather stars that have formed away from the host and were subsequently accreted.
} 

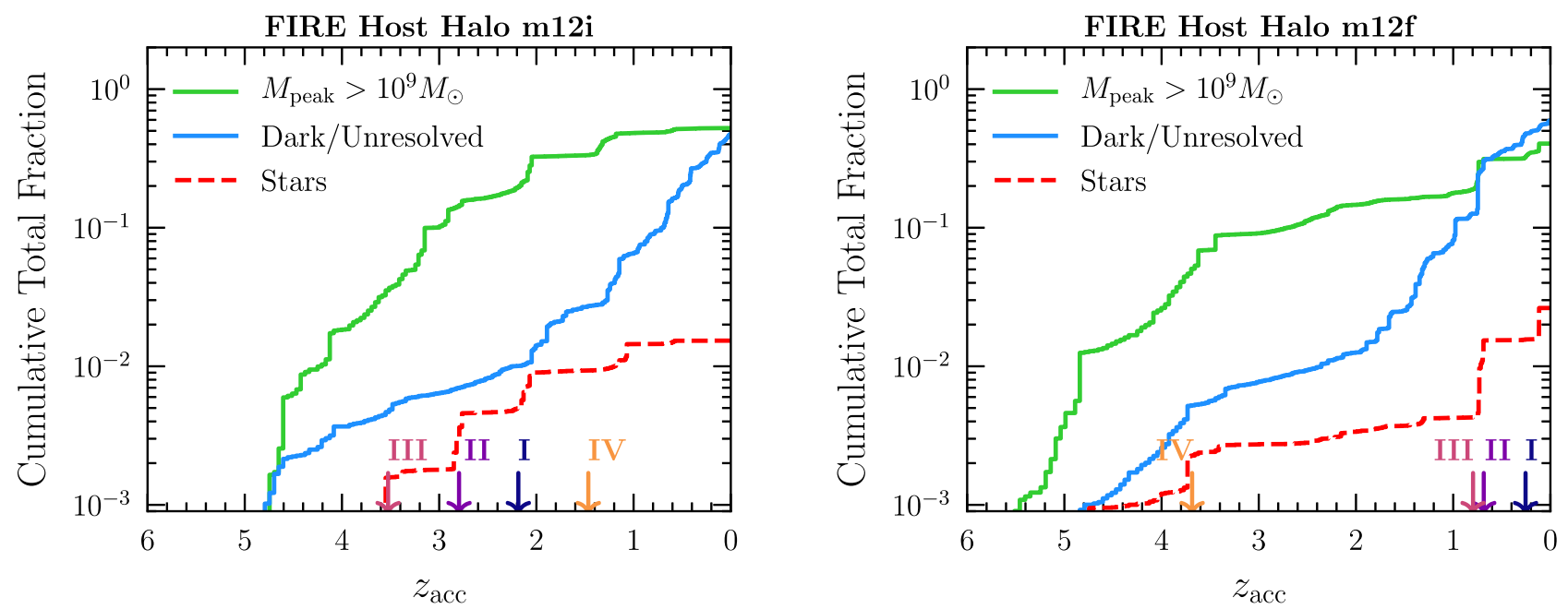

Figure 1. The cumulative fraction of dark matter and stars at the solar circle of simulated host $\mathrm{m} 12 \mathrm{i}$ (left) and $\mathrm{m} 12 \mathrm{f}$ (right). The dark matter is divided into two separate contributions. The first (green solid) is from luminous satellite galaxies with peak halo mass $M_{\text {peak }}>10^{9} M_{\odot}$. The second (aqua solid) is dark matter that originates from either a subhalo whose galaxy was not adequately resolved, a truly dark subhalo, an unresolved subhalo, or smooth accretion; due to the finite-mass resolution of the DM and star particles in the simulation, it is not possible to further distinguish its origin. The dashed red line corresponds to the cumulative fraction of accreted stars. The cumulative fraction is defined with respect to the total number of particles of each kind found in the solar circle at redshift $z=0$. The deficit below unity at $z_{\text {acc }}=0$ for the stellar distribution corresponds to its in situ fraction.

Table 1

Properties of the Top Four Mergers (Labeled I-IV) in m12i and m12f, Ranked by the Fraction of Accreted Stellar Mass Each Contributes to the Solar Circle

\begin{tabular}{|c|c|c|c|c|c|c|c|c|}
\hline & \multicolumn{4}{|c|}{ FIRE m12i Host Halo } & \multicolumn{4}{|c|}{ FIRE m12f Host Halo } \\
\hline & $\mathrm{I}$ & II & III & IV & $\mathrm{I}$ & II & III & IV \\
\hline $\begin{array}{l}M_{\text {peak }}\left[M_{\odot}\right] \\
\langle[\mathrm{Fe} / \mathrm{H}]\rangle\end{array}$ & $\begin{array}{c}3.6 \times 10^{10} \\
-1.75\end{array}$ & $\begin{array}{c}9.6 \times 10^{9} \\
-1.81\end{array}$ & $\begin{array}{c}3.4 \times 10^{10} \\
-2.01\end{array}$ & $\begin{array}{c}6.5 \times 10^{10} \\
-1.63\end{array}$ & $\begin{array}{c}1.5 \times 10^{11} \\
-0.88\end{array}$ & $\begin{array}{c}3.7 \times 10^{9} \\
-0.97\end{array}$ & $\begin{array}{c}8.2 \times 10^{10} \\
-1.16\end{array}$ & $\begin{array}{c}3.3 \times 10^{10} \\
-1.79\end{array}$ \\
\hline$M_{\text {peak }} / M_{* \text {,total }}$ & 120 & 30 & 257 & 135 & 71 & 3.4 & 57 & 180 \\
\hline Stellar mass fraction & $40 \%$ & $25 \%$ & $11 \%$ & $9 \%$ & $53 \%$ & $18 \%$ & $8.7 \%$ & $4.7 \%$ \\
\hline Dark matter mass fraction & $28 \%$ & $3 \%$ & $0.4 \%$ & $27 \%$ & $22 \%$ & $3.5 \%$ & $28 \%$ & $2.8 \%$ \\
\hline Stellar accretion redshift $\left(z_{\text {acc }}\right)$ & $2.04-2.34$ & $2.76-2.83$ & 3.53 & $1.17-1.76$ & $0.12-0.39$ & $0.68-0.70$ & $0.73-0.86$ & $3.64-3.74$ \\
\hline
\end{tabular}

Note. For each galaxy, we list the peak mass of its dark matter halo $\left(M_{\text {peak }}\right)$, average stellar metallicity $(\langle[\mathrm{Fe} / \mathrm{H}]\rangle)$, and peak halo-to-stellar mass ratio $\left(M_{\text {peak }} / M_{* \text {,total }}\right)$. The stellar mass is computed as the maximum stellar mass of the satellite over the 10 snapshots that precede its merging with the main halo. We also provide the stellar and dark matter mass fractions contributed by each satellite galaxy within the solar circle. Note that all fractions are taken with respect to the total accreted material from subhalos with $M_{\text {peak }}>10^{9} M_{\odot}$ in the simulation. The range of accretion redshifts $\left(z_{\text {acc }}\right)$ for the stars that are stripped from each satellite is also listed.

The next $25 \%$ of stars were accreted at $z_{\text {acc }}=2.76-2.83$ from a $9.6 \times 10^{9} M_{\odot}$ satellite. In contrast, the majority of the local stellar halo in $\mathrm{m} 12 \mathrm{f}$ formed at lower redshifts. For example, nearly half of the accreted stellar mass at the solar circle today was accreted between $z_{\text {acc }}=0.12-0.39$.

Because the dominant mergers in $\mathrm{m} 12 \mathrm{f}$ are typically younger relative to those of $\mathrm{m} 12 i$, they are more luminous and have a smaller ratio of peak mass to stellar mass with $M_{\text {peak }} / M_{* \text {,total }}=3.4-180^{14}$ compared to $30-257$ for m12i. This also leads to a more metal-rich population of accreted stars for $\mathrm{m} 12 \mathrm{f}$ relative to $\mathrm{m} 12 \mathrm{i}$, with mean metallicities of the

\footnotetext{
14 There are instances when the algorithm cannot locate a satellite at a given snapshot when tracing it back in time. This can potentially affect the values of $M_{\text {peak }} / M_{* \text {,total }}$ quoted in Table 1 . This issue is exacerbated if the merger is particularly violent, with the satellite mass changing substantially from snapshot to snapshot (thereby making it difficult for the merger tree to recognize that it is the same object). Merger II of $\mathrm{m} 12 \mathrm{f}$ is one such example, so its estimated $M_{\text {peak }} / M_{* \text {,total }}$ should be considered with care. Merger III of $\mathrm{m} 12 \mathrm{i}$ is another case, though less pronounced.
}

dominant mergers closer to $\langle[\mathrm{Fe} / \mathrm{H}]\rangle_{\mathrm{m} 12 \mathrm{f}} \sim-1.2$ compared to $\langle[\mathrm{Fe} / \mathrm{H}]\rangle_{\mathrm{m} 12 \mathrm{i}} \sim-1.8$.

Mergers I-IV contribute nearly all of the local accreted stellar mass in $\mathrm{m} 12 \mathrm{i}$ and $\mathrm{m} 12 \mathrm{f}$, and a majority of the accreted DM. "Accreted DM" refers to the DM that originates from subhalos with $M_{\text {peak }}>10^{9} M_{\odot}$, and excludes the "Dark/ Unresolved" component. In $\mathrm{m} 12 i$, for example, $85 \%$ of the accreted stellar mass comes from Mergers I-IV, whereas 58\% of the accreted DM does. In m12f, the top four mergers contribute $84 \%$ of the accreted stars and $56 \%$ of the accreted DM.

Figure 1 shows the cumulative fraction of DM as a function of accretion redshift for $\mathrm{m} 12 \mathrm{i}$ (left) and $\mathrm{m} 12 \mathrm{f}$ (right). We separately show the total DM that was accreted from galaxies with $M_{\text {peak }}>10^{9} M_{\odot}$ in green and the "Dark/Unresolved" component in aqua. As discussed in Section 2, $M_{\text {peak }} \sim 10^{9} M_{\odot}$ is roughly the lower limit for luminous satellites in the simulation given the resolved star particle mass. Luminous satellites in the simulation with halo masses above this limit 
FIRE Host Halo m12f
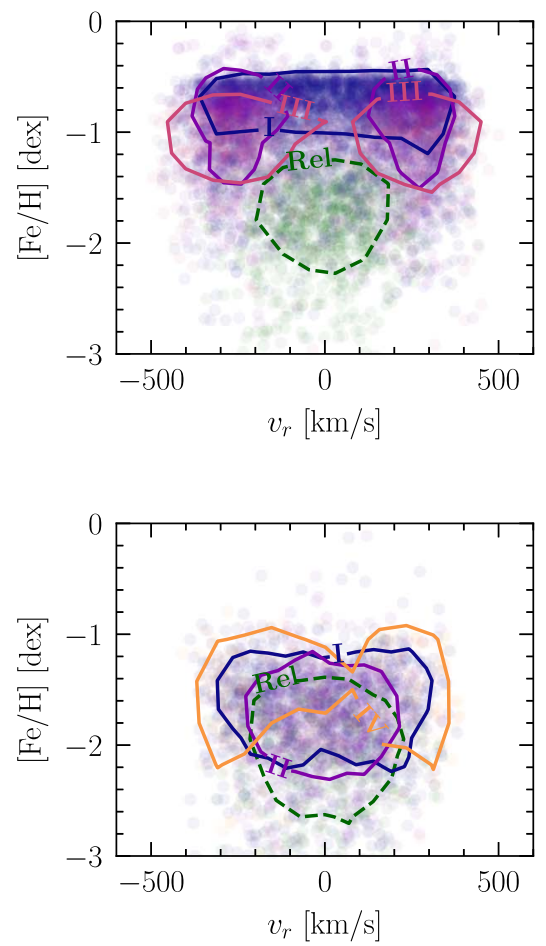

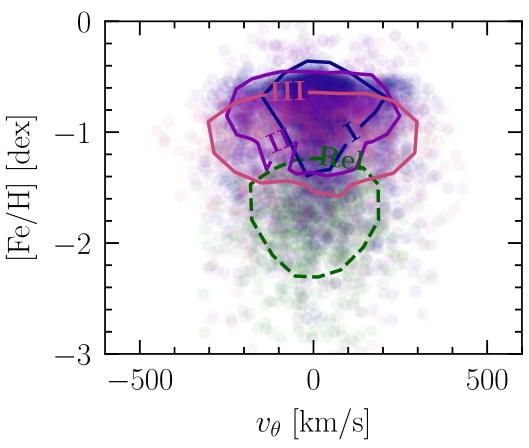

FIRE Host Halo m12i

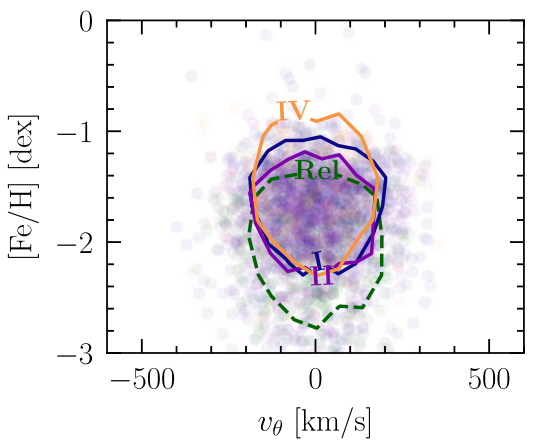

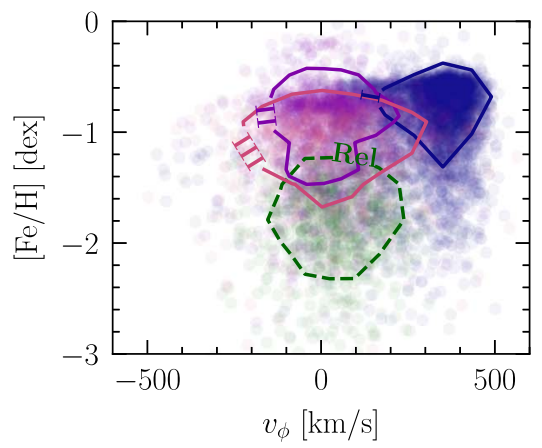

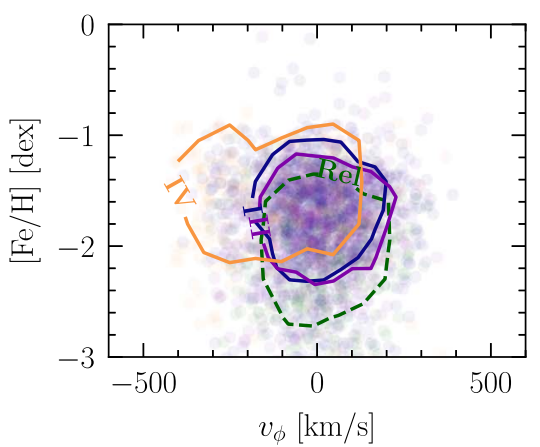

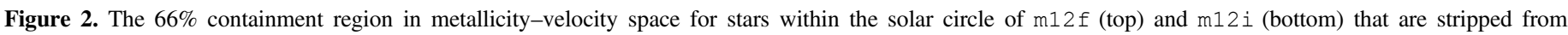

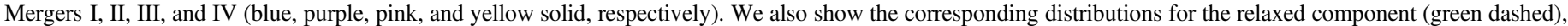

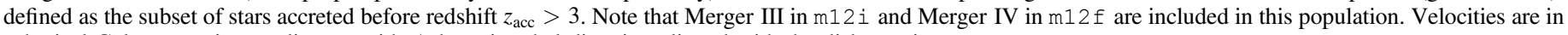
spherical Galactocentric coordinates, with $\phi$ the azimuthal direction aligned with the disk rotation.

offer an opportunity to compare the final positions of accreted stars and DM.

Figure 1 shows the cumulative fraction of the stars accreted from these satellites in dashed red. The distinct steps in the cumulative stellar fraction occur at the average $z_{\text {acc }}$ for stars stripped from Mergers I-IV (indicated by the arrows in the figure). Similar steps are observed in the cumulative DM fraction at roughly the same redshifts. This explicitly demonstrates that the mergers dragged in significant amounts of both DM and stars to the solar circle at approximately the same times.

The fact that the jumps in the DM cumulative fraction closely align with those in the stars suggests that it is the mostbound DM of each satellite that contributes at the solar circle. In general, we expect that tides start to remove DM from a satellite earlier than its stars because the halo is more extended. By the time the satellite's orbit sinks down to the inner parts of the galaxy; however, most of its DM halo has been stripped off, leaving behind only the most-bound portion. In this respect, the Sun's location at the inner galaxy is fortuitous for reconstructing the DM velocities from stellar orbits.

\section{Correlations between Accreted Stars and Dark Matter}

The phase-space distribution of the DM and stars within the solar circle is intimately linked with the galaxy's accretion history. DM and stars that accreted onto the host at early epochs $\left(z_{\text {acc }} \gtrsim 3\right)$ are fully relaxed. More recent accretion events, however, continue to build up the local mass profile. If this debris is not fully phase-mixed, it can be identified as substructure in either position or velocity space.
Figure 2 demonstrates how the stars in both the relaxed and substructure populations cluster in metallicity-velocity space. In general, elemental abundances provide an important handle when linking stellar debris to a progenitor galaxy (Johnston et al. 1995, 1996; Helmi \& White 1999; Bullock et al. 2001; Bullock \& Johnston 2005; Purcell et al. 2007; De Lucia \& Helmi 2008); we focus on the iron abundance $[\mathrm{Fe} / \mathrm{H}]$ here. Figure 2 shows the distributions of $[\mathrm{Fe} / \mathrm{H}]$ against $v_{r}, v_{\theta}, v_{\phi}$ for stellar debris of m12f (top) and m12i (bottom). Note that we use spherical galactocentric velocities throughout, with $\phi$ oriented with the disk rotation. The relaxed stellar component is shown in green, while the stellar populations associated with Mergers I, II, III, and IV are shown in blue, purple, pink, and yellow, respectively. Merger III of $\mathrm{m} 12 i$ and Merger IV of m12 $\mathrm{f}$ are included in their respective relaxed populations. Clearly, a wide variety of kinematic features are possible. While the relaxed stellar population appears to be nearly isotropic, the more recent mergers exhibit distinctive kinematic features. Taken together, the chemical abundance and kinematics of stellar populations can play an important role in identifying their origin.

In this section, we explore in detail the phase-space evolution of DM and stars from mergers in m12i and m12 $f$. We systematically study the contributions to the solar circle, from the oldest to the youngest accreted material. In this way, we will see how the velocity distribution of the accreted stars is built up as a function of time, and how well it traces the DM as the two evolve and grow together. Host halo m12f provides a contrasting example to $\mathrm{m} 12 i$, because its merger history is more active up until redshift $z \sim 0.1$. 
FIRE Host Halo m12f, Relaxed Component
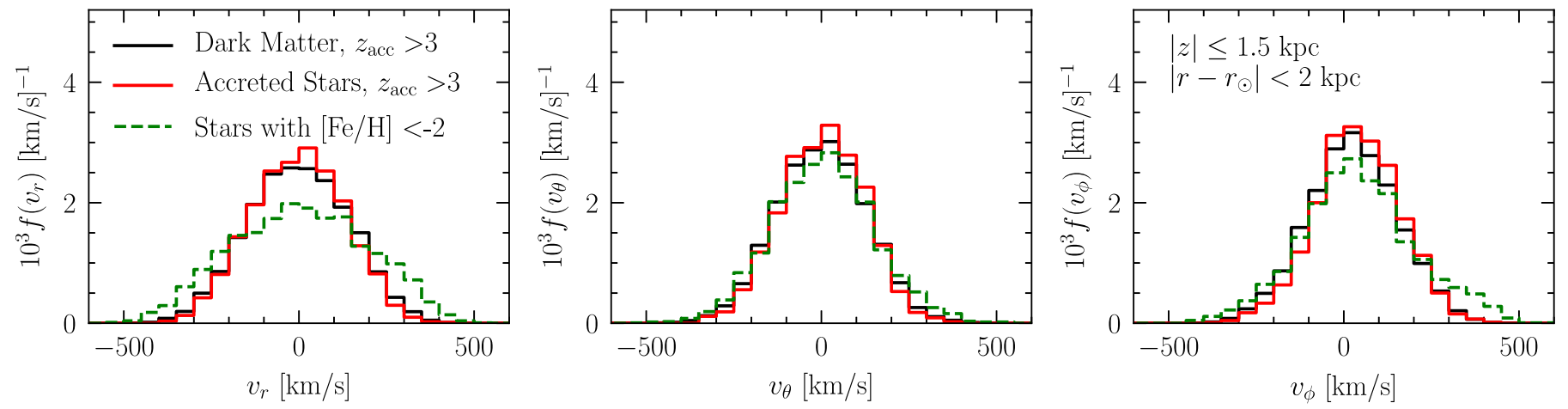

FIRE Host Halo m12i, Relaxed Component
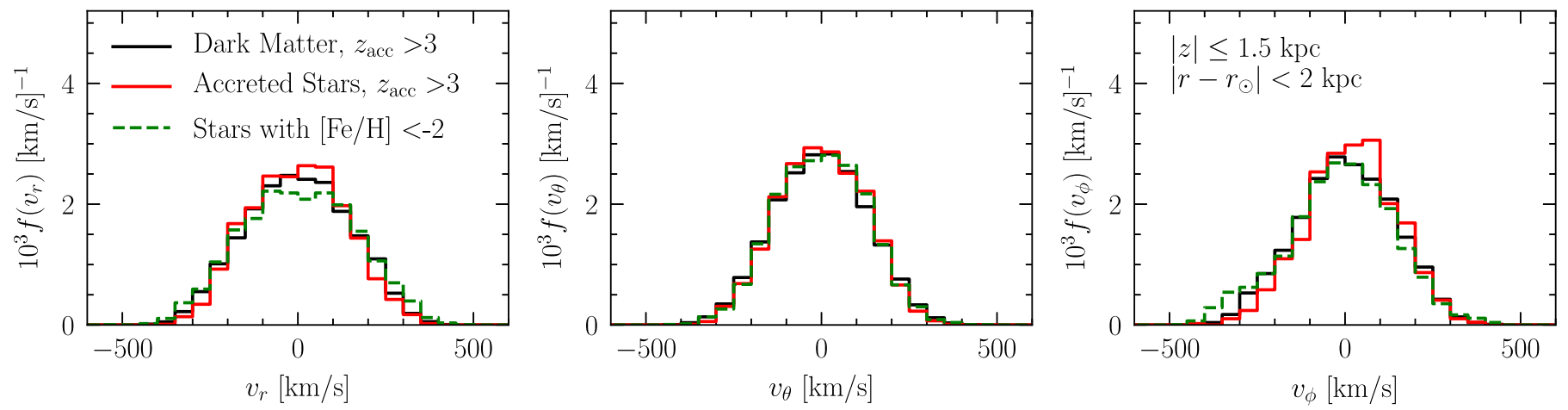

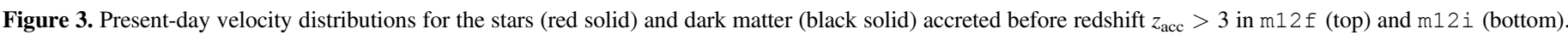

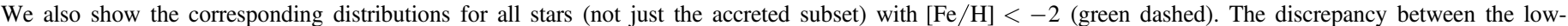

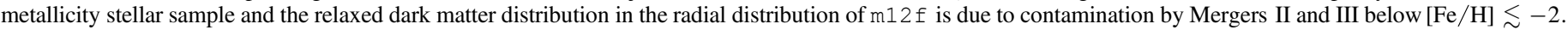

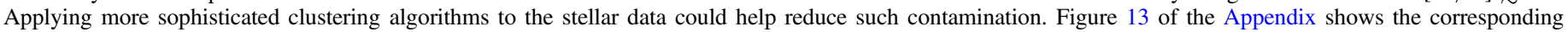
distributions for $[\mathrm{Fe} / \mathrm{H}] \lesssim-3$.

The results of this section pertain specifically to DM that is sourced by luminous satellites $\left(M_{\text {peak }} \gtrsim 10^{9} M_{\odot}\right)$. The kinematic distributions of DM from the "Dark/Unresolved" component is discussed in Section 5.2.

\subsection{The Relaxed Component}

Violent relaxation plays an important role in mixing stars and DM that accreted from a galaxy's oldest mergers. Nonadiabatic transformations of the potential change the energies of the stars and DM, causing their orbits to fill the available phase space. These effects are particularly important in the period when the proto-Milky Way is forming. This process is distinct from changes to the course-grained phasespace distribution that arise as a system evolves in time following Liouville's theorem. In this process, both the original phase-space volume and energy are conserved as time evolves. This phase-mixing process drives the evolution of streams and debris flow, as described in Section 4.2.

We begin by focusing on the present-day distribution of stars and DM in m12i and $\mathrm{m} 12 \mathrm{f}$ that were accreted from the earliest mergers $\left(z_{\text {acc }}>3\right)$. There are 21 significant mergers that contribute to this population in $\mathrm{m} 12 \mathrm{i}$, and 39 for $\mathrm{m} 12 \mathrm{f}$. Note that the relaxed population in m12i includes Merger III, and in $\mathrm{m} 12 \mathrm{f}$ includes Merger IV. The average metallicity of the stars in the relaxed component is $\langle[\mathrm{Fe} / \mathrm{H}]\rangle_{\mathrm{m} 12 \mathrm{i}}=-2.13$ $(0.53$ dex spread $)$ for $\mathrm{m} 12 i$, and $\langle[\mathrm{Fe} / \mathrm{H}]\rangle_{\mathrm{m} 12 \mathrm{f}}=-1.88$ (0.45 dex spread) for m12f.
The velocity distributions of the relaxed stellar component in $\mathrm{m} 12 i$ is indicated by the red lines in the bottom panel of Figure 3. The distributions are approximately isotropic, with dispersions of $\left\{\sigma_{r}, \sigma_{\theta}, \sigma_{\phi}\right\}=\{136,122,125\} \mathrm{km} \mathrm{s}^{-1}$. Notably, the stellar and DM distributions, which are indicated in black, trace each other closely. The discrepancies between the two are small, ranging from $1 \%$ to $16 \%$ in any given bin, but closer to $\sim 50 \%$ along the tails. As the top panel of Figure 3 shows, these results are similar for m12f. To estimate the statistical uncertainties, we bootstrapped by subsampling the DM to match the size of the stellar sample. We found that there can be $\sim 70 \%$ statistical uncertainties in the ratio of the stellarto-DM distribution in the highest velocity bins with $\gtrsim \pm 350 \mathrm{~km} \mathrm{~s}^{-1}$.

Using the ERIS simulation, Herzog-Arbeitman et al. (2018a) demonstrated that metal-poor stars act as kinematic tracers for the relaxed DM component. ${ }^{15}$ To test whether the same results are reproduced with FIRE, we compare the relaxed distributions to those of all stars (not just the accreted subset) with a metallicity cut of $[\mathrm{Fe} / \mathrm{H}]<-2$ (green dashed). For m12i, the metal-poor stars trace the relaxed component of DM and stars almost exactly. The correspondence for $\min \mathrm{f}$ is also very

\footnotetext{
${ }^{15}$ Note that what we refer to as "relaxed" here is referred to as "virialized" in Herzog-Arbeitman et al. (2018a). The ERIS study did not break down the DM into components from older versus more recent mergers. The fact that a good correspondence was already observed between DM and metal-poor stars is due to the quiet merger history of ERIS, as its last major merger occurred at redshift $z \sim 3$.
} 
FIRE Host Halo m12f, Relaxed Component
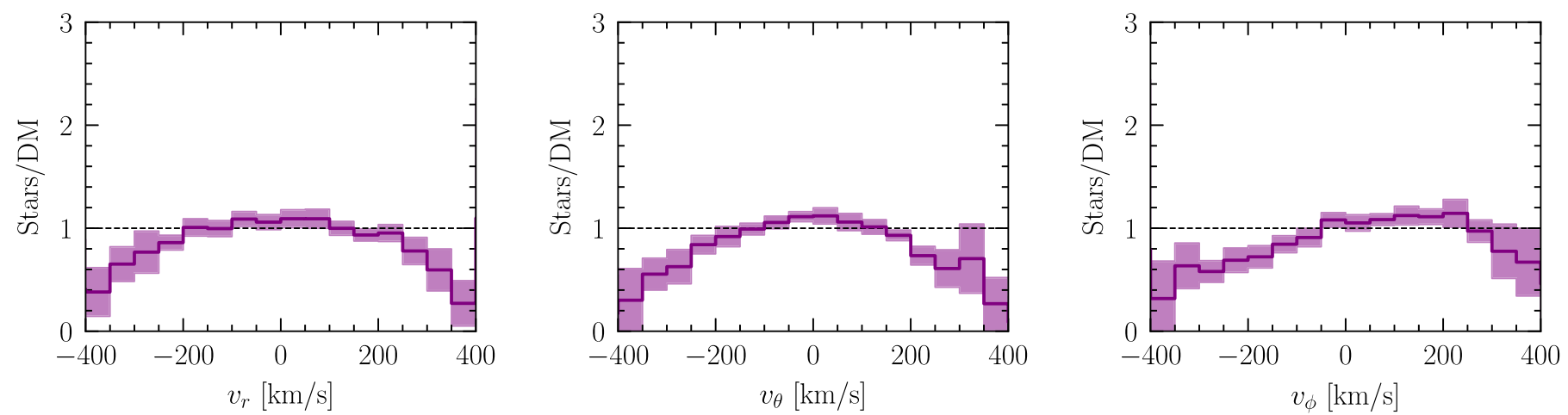

FIRE Host Halo m12i, Relaxed Component
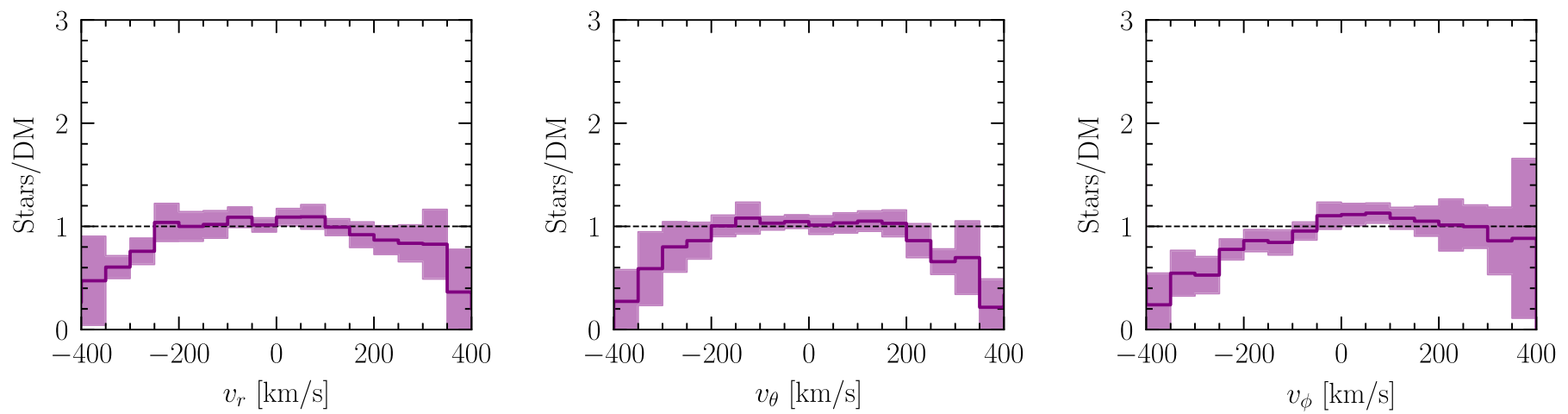

Figure 4. The ratio of the stellar-to-dark matter (DM) velocity distributions for the relaxed population of m12f (top) and $\mathrm{m} 12 \mathrm{i}$ (bottom). Results are shown separately for the separate Galactocentric velocity components. The distributions are sampled in 10 locations throughout the solar circle, within spheres of radius 4 kpc centered at a Galactic distance of $r_{\odot}=8 \mathrm{kpc}$. The mean ratio over these regions is indicated by the solid purple line, and the colored band indicates the $1 \sigma$ spread.

good, especially for $v_{\theta}$ and $v_{\phi}$. For the radial distribution, the distribution of metal-poor stars is clearly more extended. This arises from the contamination of the high-radial velocity lobes of Mergers II and III, which extend below $[\mathrm{Fe} / \mathrm{H}]<-2$ (see Figure 2). Tightening the metallicity requirement to $[\mathrm{Fe} / \mathrm{H}]<-3$ brings the metal-poor distributions even more in line with the relaxed distributions-see Figure 13 of the Appendix.

In practice, it is possible to reduce the contamination of more recent mergers, such as Mergers II and III of $\mathrm{m} 12 \mathrm{f}$, from the reconstructed distributions of the relaxed population by using a more sophisticated clustering algorithm, such as that performed in Necib et al. (2019) which can group stars based both on their metallicities and velocities. Applied to the local stellar halo of m12f, for example, such a procedure could potentially distinguish the stars with $[\mathrm{Fe} / \mathrm{H}]<-2$ that are kinematically more similar to Mergers II and III versus the relaxed population.

Figure 4 shows how the ratio of the relaxed stellar-to-DM velocity distributions varies across the solar circle. We sample the stars and DM in spheres of radius $4 \mathrm{kpc}$ that are centered at a Galactic distance of $r_{\odot}=8 \mathrm{kpc}$. The solid purple line in Figure 4 denotes the mean value over 10 sampled locations, and the band indicates the $1 \sigma$ spread. For each velocity component, the mean is consistent with unity over the majority of the velocity range, with a small overall spread between regions. Discrepancies are typically $\lesssim 20 \%$ over all regions, but increase to $\sim 50 \%$ in the highest velocity bins, where we also find large statistical uncertainties. These results underline the fact that the DM-stellar correlation observed for the relaxed population is mostly consistent in localized regions throughout the solar circle. It is important, however, to point out that direct detection limits for light $(\sim 10 \mathrm{GeV})$ DM scattering off nuclei are most sensitive to the tails of the distributions, where we observe the largest discrepancies in the velocity correlations.

\subsection{Substructure Component}

After a host galaxy's last major merger, its potential changes adiabatically as DM and stars continue to be accreted through relatively smaller mergers. The material that is stripped is initially confined to a small region in phase space, but it evolves with time to eventually become fully mixed. The observable features of this debris depend on the elapsed time since the merger. For example, when the time $t$ since accretion is on the order of the dynamical time of the system $\left(t \sim t_{\mathrm{dyn}}\right)$, the remains of a disrupted satellite are not well mixed either spatially or kinematically and manifest as a stream, a structure that is dynamically cold and typically coherent in speed. Stellar streams have been observed throughout the Milky Way halosee Grillmair \& Carlin (2016) and references therein - with the most studied example coming from Sagittarius (Ivezić et al. 2000; Yanny et al. 2000). DM streams have been studied in numerous $N$-body simulations (Diemand et al. 2008; Vogelsberger et al. 2009; Zemp et al. 2009; Kuhlen et al. 2010; Elahi et al. 2011; Maciejewski et al. 2011; Vogelsberger \& White 2011). 
FIRE Host Halo m12f Merger I
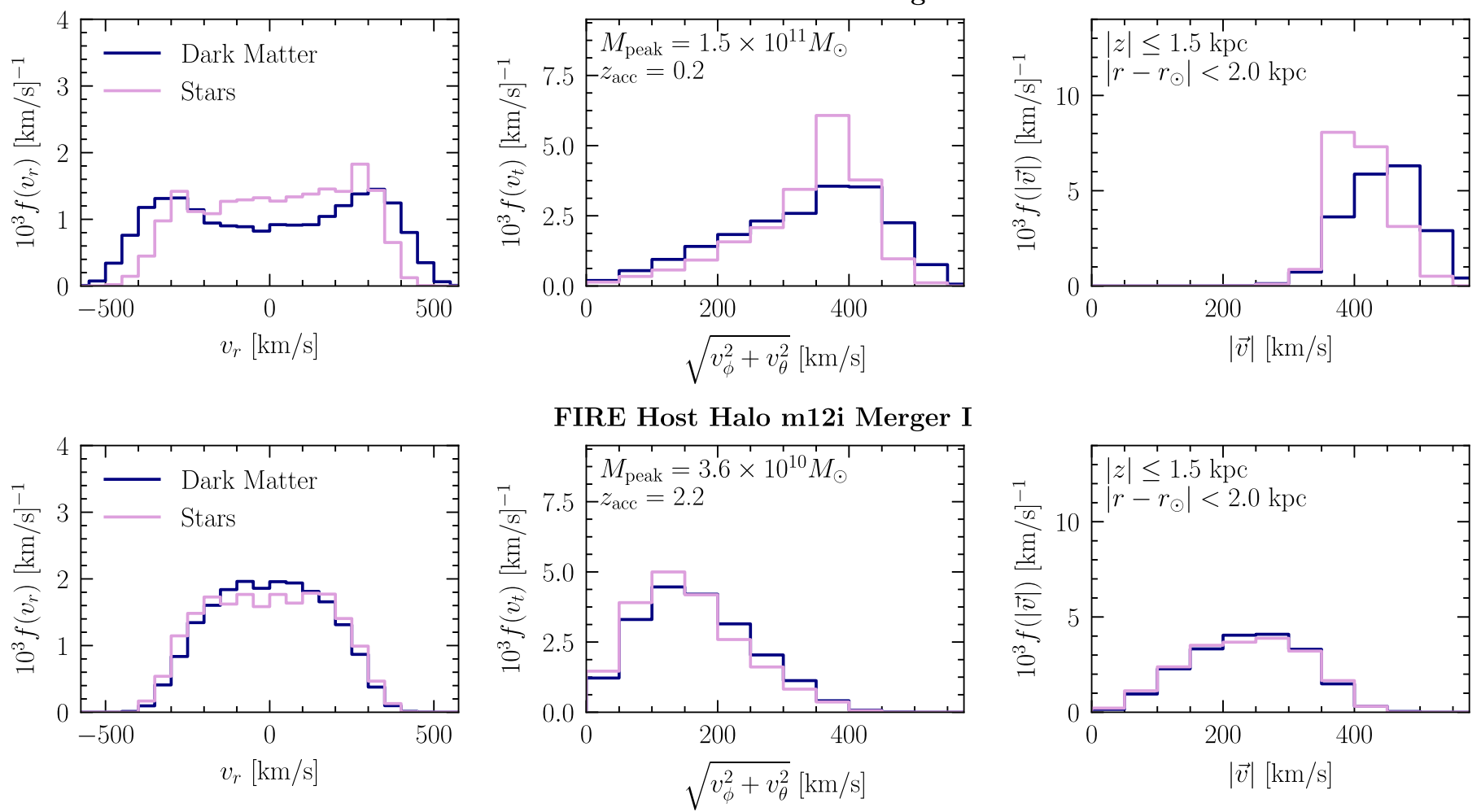

Figure 5. Present-day velocity distributions for the debris of Merger I of $\mathrm{m} 12 \mathrm{f}$ (top) and Merger II of $\mathrm{m} 12 \mathrm{i}$ (bottom) that falls within the solar circle. The radial (left), tangential (middle), and speed (right) distributions are shown for the stars (purple solid) and dark matter (blue solid). The details of the mergers are provided in Table 1; the corresponding distributions for the other mergers listed in the table are provided in Figures 14 and 15 of the Appendix. As discussed in the text, Merger I of $\mathrm{m} 12 \mathrm{f}$ is an example of a stream, while Merger I of m12 $i$ is an example of debris flow.

The most significant merger within the solar circle of m12f leaves behind a stream. The top row of Figure 5 shows the radial and tangential velocity distributions, as well as the speed distribution, for the DM and stars from this merger. The stellar distribution (purple) is broad in the radial direction, while its tangential distribution is peaked at $\sim 400 \mathrm{~km} \mathrm{~s}^{-1}$. The stars are reasonably coherent in speed, as demonstrated in the rightmost panel. The corresponding DM distributions are shown in blue. While the DM and stellar kinematics share similar features, they do not trace each other exactly. For example, the discrepancies between the stellar and DM speed distributions are within $3 \%-80 \%$, but reach a factor of $\sim 2-4$ at the tails.

The top panel of Figure 6 shows the spatial distribution of the stars (left) and DM (right) from Merger I of m $12 \mathrm{f}$. The stars are clustered around $x \sim 10 \mathrm{kpc}$ along the midplane. Their spatial distribution is distinct from that of the DM, which is more uniformly distributed although still clustered in the midplane. The fact that the stars and DM have different spatial distributions results in large local variations in their kinematic distributions. The top panel of Figure 7 shows how the ratio of the stellar-to-DM velocity distributions varies across the solar circle. On average, the ratio of the stellar and DM distributions is unity, but the spread is quite large-reaching discrepancies of $\gtrsim 2$ in certain locations. The discrepancies are particularly pronounced in the speed distribution.

As time proceeds $\left(t>t_{\mathrm{dyn}}\right)$, the velocity dispersion of any individual stream decreases as the stars spread out in position space following Liouville's theorem (Helmi \& White 1999). Debris flow (Kuhlen et al. 2012; Lisanti \& Spergel 2012;
Lisanti et al. 2015) consists of multiple wraps of these streams, as well as any shells that formed in the process of satellite disruption. While these contributions are individually cold, their sum is dynamically hot. ${ }^{16}$ Debris flow is therefore the intermediate state of tidal debris before it becomes fully mixed with the host halo at $t \gg t_{\mathrm{dyn}}$. It is identified as kinematic substructure that is coherent over large spatial regions.

Merger I of m12i, whose velocity distributions are provided in the bottom panel of Figure 5, is an example of debris flow. The stellar material from this satellite was accreted at $z_{\text {acc }} \sim 2.2$ and is therefore older than Merger I of m12f. In this case, the DM and stars trace each other closely in all velocity components. The deviations between the distributions are typically under $18 \%$ in each bin, reaching $~ 35 \%$ in some bins along the tails. Additionally, the DM and stellar debris from this merger are spatially uniform within the solar circle, as shown in the bottom panel of Figure 6.

The velocity distribution of the stars and DM of m12i's Merger I retain important features that correspond to the satellite's orbital properties, even if the sharp coherence in speed is lost. For example, the radial velocity distribution is extended and box-like, a feature of satellites on radial orbits. In such cases, most of the debris is stripped as the satellite moves toward/away from the galactic center, resulting in two peaks of the same radial speed, but with opposite directions $\left( \pm v_{r}\right)$. If the dispersion of these peaks is considerably larger than $v_{r}$, then

\footnotetext{
${ }^{16}$ We also note that debris flow may arise from more than one disrupted satellite if the two happened to be on similar orbits and were accreted at comparable times.
} 
FIRE Host Halo m12f Merger I
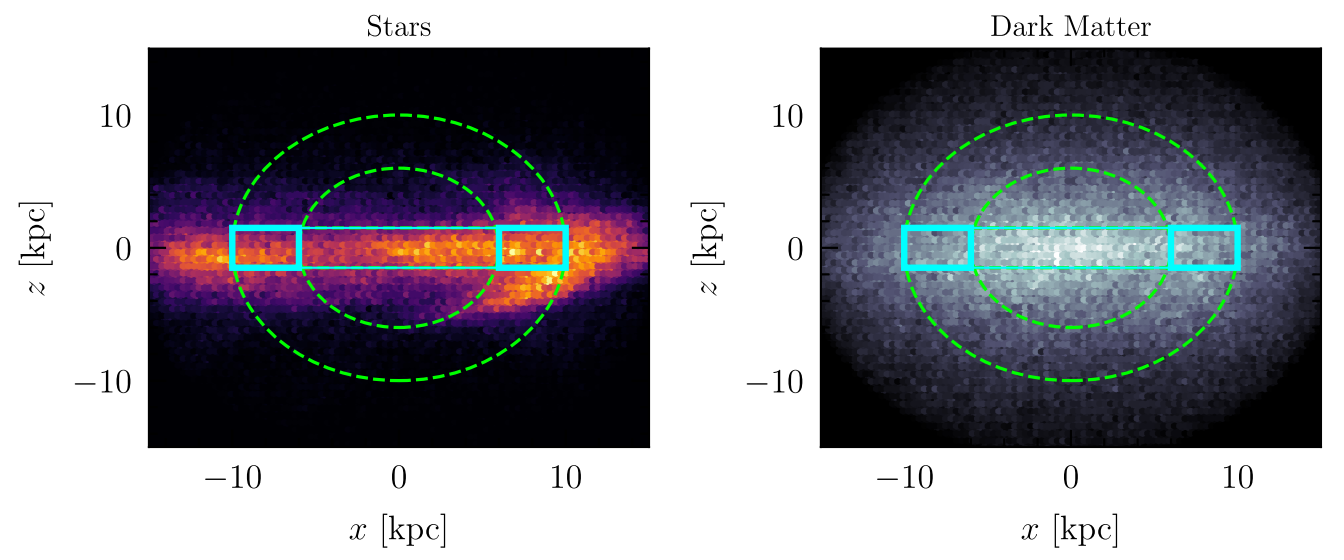

FIRE Host Halo m12i Merger I
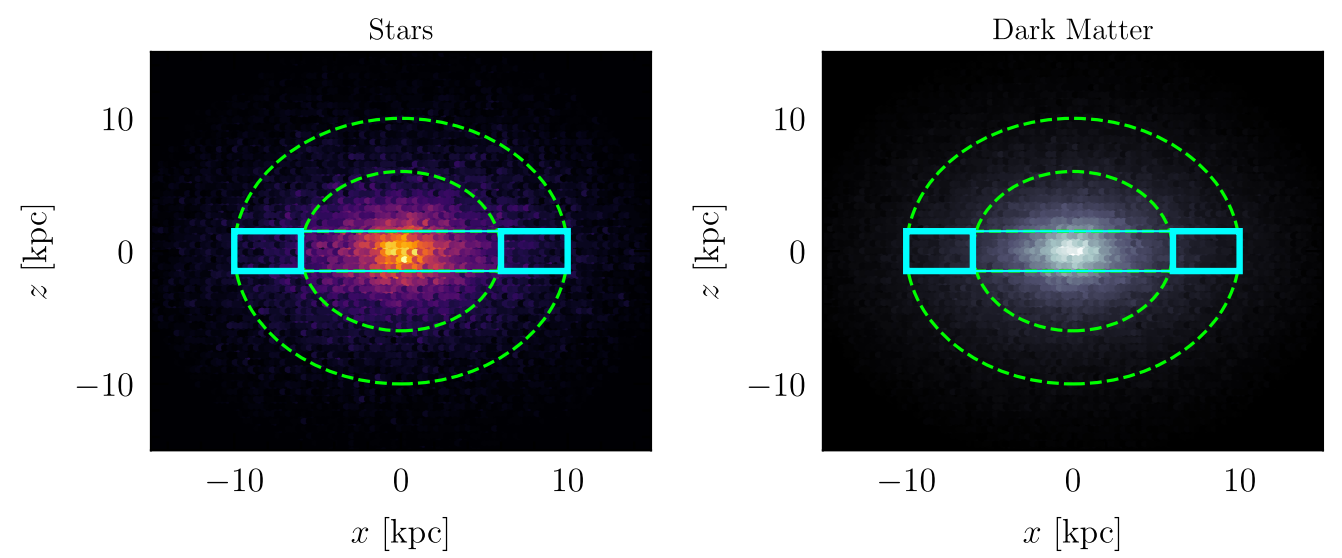

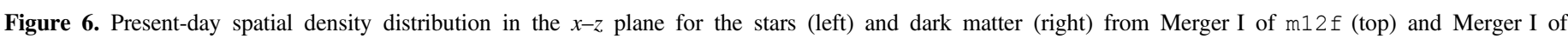

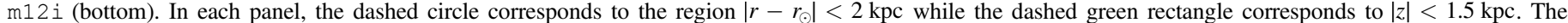
intersection of these two regions, denoted by the solid blue rectangle, is the solar circle.

they bleed into each other, forming a box-like distribution. This is expected if the turning points of the orbit do not fall near or within the solar circle, so one is primarily sampling material that is removed while the satellite is on a radial trajectory.

Because the spatial variation of the DM and stars is uniform in this case, their velocity distributions are consistent across localized regions of the solar circle. The bottom panel of Figure 7 shows the ratio of DM to stellar velocity distributions for this merger. In this case, the ratio is tightly centered about unity over all the regions sampled.

While we only discussed Merger I of m12 f and Merger I of m12 $i$ in this subsection, the conclusions remain unchanged when studying the other significant mergers in both hosts. The DM and stellar velocity distributions for these mergers are provided in Figures 14 and 15 of the Appendix.

\section{The Total Dark Matter Distribution}

In the previous section, we saw that the kinematics of the DM and stars accreted from luminous satellites are well correlated for older mergers, specifically, the relaxed component and debris flow. In this section, we will describe how to combine the separate contributions from these populations with the goal of constructing the DM speed distribution at the solar circle. Section 5.1 will focus on summing the contributions from the relaxed DM with that originating from Mergers I and
II in m12i. As we will see in Section 6, this methodology will have important applications for the Milky Way, given its similarities to m12i. Section 5.2 will discuss the "Dark/ Unresolved" DM component.

\subsection{Component from Luminous Satellites}

Taking m12 $i$ as an example, let us consider the scenario where the local stellar halo is dominated by two large mergers (e.g., Mergers I and II) in addition to a relaxed stellar component. The speed distributions for each of these stellar populations is $f_{\mathrm{I}}(v), f_{\mathrm{II}}(v)$, and $f_{\mathrm{r}}(v)$, respectively, with each normalized to unity. The total stellar distribution is therefore given by

$$
f_{\text {stellar }}(v)=\xi_{*, \mathrm{r}} f_{\mathrm{r}}(v)+\xi_{*, \mathrm{I}} f_{\mathrm{I}}(v)+\xi_{*, \mathrm{II}} f_{\mathrm{II}}(v),
$$

where the $\xi_{*}$ are the observed stellar mass fractions for the components and $\xi_{*, \mathrm{r}}+\xi_{*, \mathrm{I}}+\xi_{*, \mathrm{II}}=1$. These values are provided in the first row of Table 2 . Note that we have renormalized the values under the assumption that all of the accreted stars belong to either Merger I, II, or the relaxed population, to simplify the discussion.

The leftmost panel of Figure 8 shows the stacked speed distributions for the stars associated with the relaxed component (green solid), Merger I (blue solid), and Merger II 
FIRE Host Halo m12f Merger I
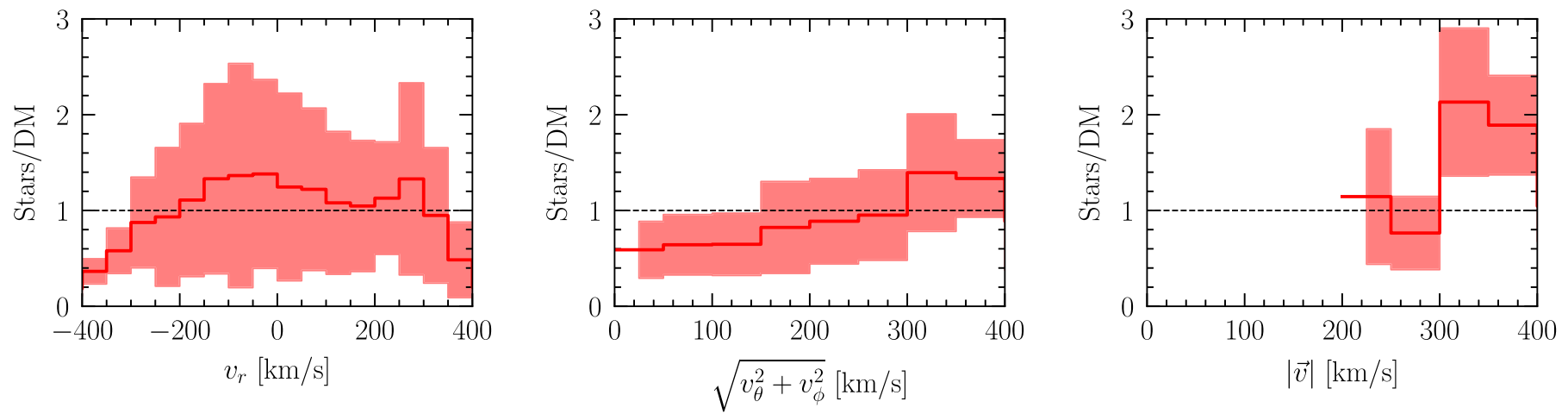

FIRE Host Halo m12i Merger I
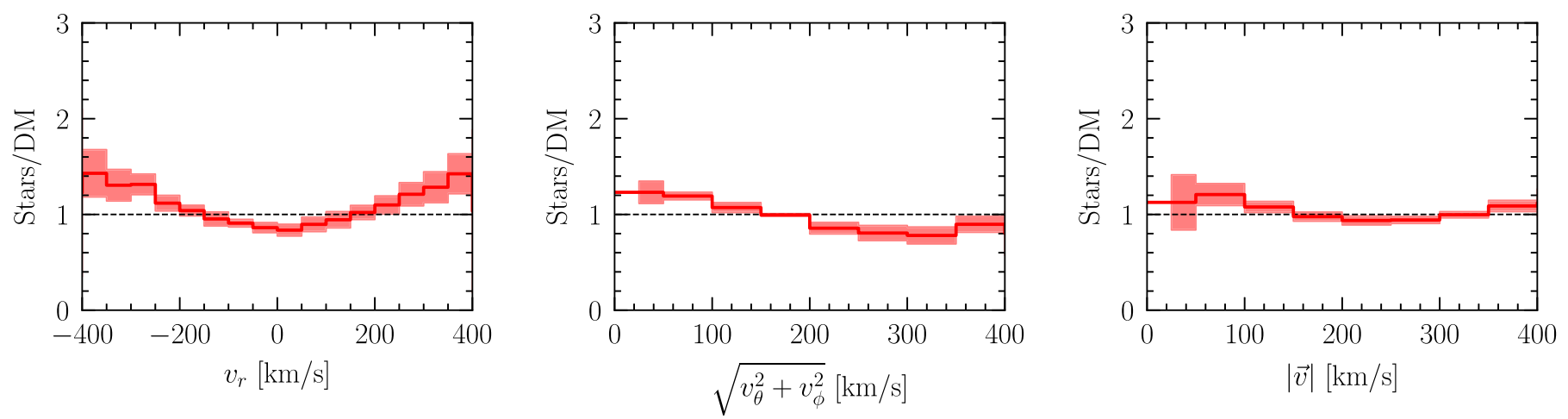

Figure 7. Same as Figure 4, except for the radial, tangential, and speed distributions of Merger I of m12f (top) and Merger I of $\mathrm{m} 12 \mathrm{i}$ (bottom).

Table 2

Relevant Fractions at the Solar Circle for the m12i and m12f Host Halos, Normalized by the Sum of the Relaxed Population and Mergers I-II

\begin{tabular}{|c|c|c|c|c|c|c|}
\hline & \multicolumn{3}{|c|}{ FIRE m12i Host Halo } & \multicolumn{3}{|c|}{ FIRE m12f Host Halo } \\
\hline & Relaxed & I & II & Relaxed & I & II \\
\hline Stellar fraction at solar circle & 0.16 & 0.52 & 0.32 & 0.17 & 0.62 & 0.21 \\
\hline Dark matter mass fraction & 0.39 & 0.55 & 0.06 & 0.46 & 0.46 & 0.07 \\
\hline$\langle[\mathrm{Fe} / \mathrm{H}]\rangle$ & -2.13 & -1.75 & -1.81 & -1.88 & -0.88 & -0.97 \\
\hline$M_{\mathrm{DM}} / M_{*}$ at solar circle & 76 & 43 & 8 & 44 & 8 & 4 \\
\hline True $M_{\text {peak }} / M_{* \text {,total }}$ & 876 & 120 & 30 & 665 & 71 & 3.4 \\
\hline Inferred $M_{\text {peak }} / M_{*, \text { total }}$ & 159 & 106 & 113 & 155 & 28 & 33 \\
\hline True $c_{i} / c_{\mathrm{r}}$ & $\ldots$ & 0.57 & 0.11 & $\ldots$ & 0.18 & 0.09 \\
\hline Inferred $c_{i} / c_{\mathrm{r}}$ & $\cdots$ & 0.67 & 0.71 & $\cdots$ & 0.18 & 0.21 \\
\hline
\end{tabular}

Note. Note that Merger III is included in the relaxed component for $\mathrm{m} 12 \mathrm{i}$ and Merger IV for $\mathrm{m} 12 \mathrm{f}$. From top to bottom, we provide the following: (i) the stellar mass from each component at the solar circle assuming only the relaxed component and Mergers I-II, (ii) the dark matter mass from each component, relative to the total accreted dark matter mass at the solar circle from the relaxed component and Mergers I-II, (iii) the mean metallicity of each component, (iv) the dark matter mass from each component, relative to its stellar mass at the solar circle, (v) the true $M_{\text {peak }} / M_{* \text {,total }}$ from the simulation, (vi) the inferred $M_{\text {peak }} / M_{* \text {,total }}$ from the procedure described in the text (using Equation (4) for m12i for example), (vii) the true $c_{i} / c_{\mathrm{r}}\left(i=\mathrm{I}\right.$ or II) values, and (vii) the inferred $c_{i} / c_{\mathrm{r}}$ values using the estimated mass-tolight ratio.

(purple solid), combined according to Equation (1). This corresponds to the total speed distribution for the accreted stars. Let us compare this to the stacked distributions for the DM associated with these same populations (shown in gray). Clearly, the two do not match. We have already seen that the stellar distributions for the separate populations of $\mathrm{m} 12 i$ reproduce those of the DM (see Figures 3, 5, and 14). Therefore, the source of the discrepancy arises from using the stellar mass fractions in Equation (1).
To reproduce the total DM distribution, we should instead use the DM mass fraction $\xi_{\mathrm{dm}}$ for each component as its appropriate weight in the sum:

$$
f_{\mathrm{dm}}(v)=\xi_{\mathrm{dm}, \mathrm{r}} f_{\mathrm{r}}(v)+\xi_{\mathrm{dm}, \mathrm{I}} f_{\mathrm{I}}(v)+\xi_{\mathrm{dm}, \mathrm{II}} f_{\mathrm{II}}(v) .
$$

The $\xi_{\mathrm{dm}}$ values are provided in the second row of Table 2 . Using these exact weights, we can stack the stellar distributions according to Equation (2); the result is shown in the middle 

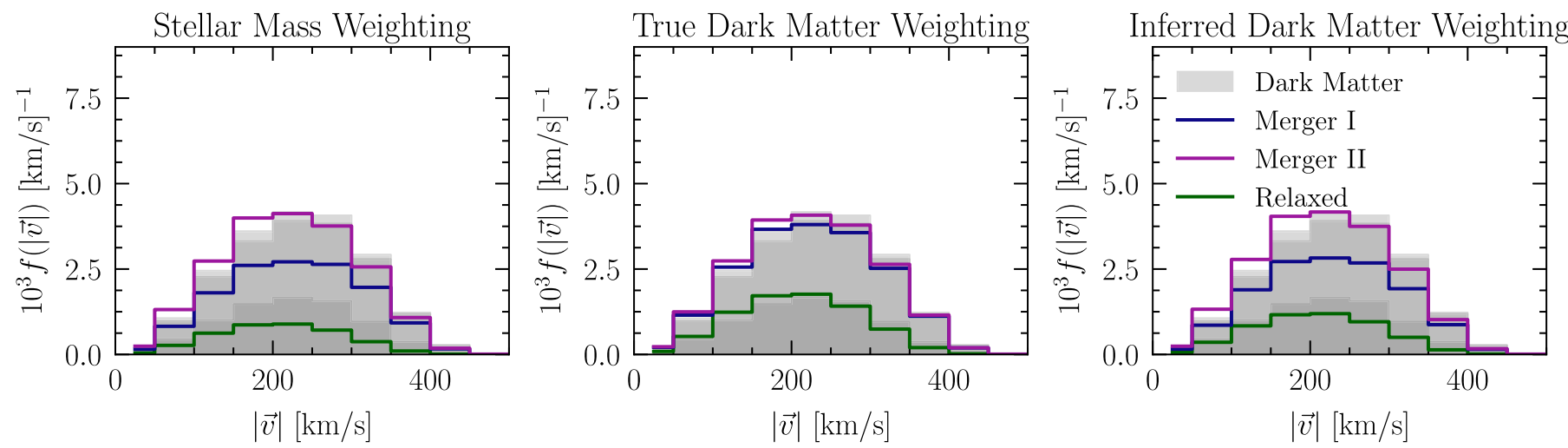

Figure 8. Reconstructing the speed distribution of dark matter from the accreted stars of $\mathrm{m} 12 i$. The true dark matter distributions for the relaxed component and from Mergers I and II are stacked from bottom to top in gray. The distributions inferred from the corresponding stellar populations are shown by the colored lines (green, blue, and purple, respectively). To add the stellar speed distributions, we (left) use the stellar mass fractions as per Equation (1), (middle) follow Equation (2) and take the exact values of the dark matter mass fractions, and (right) follow Equation (3) and take the inferred values of $c_{i} / c_{r}$ from the mass-to-light ratios. A similar plot for $\mathrm{m} 12 \mathrm{f}$ is provided in the Appendix as Figure 16. As described in the text, the remaining discrepancy in the total distributions in the right panel is due to the uncertainty in extrapolating the mass-to-light ratios for the individual mergers.

FIRE Host Halo m12i
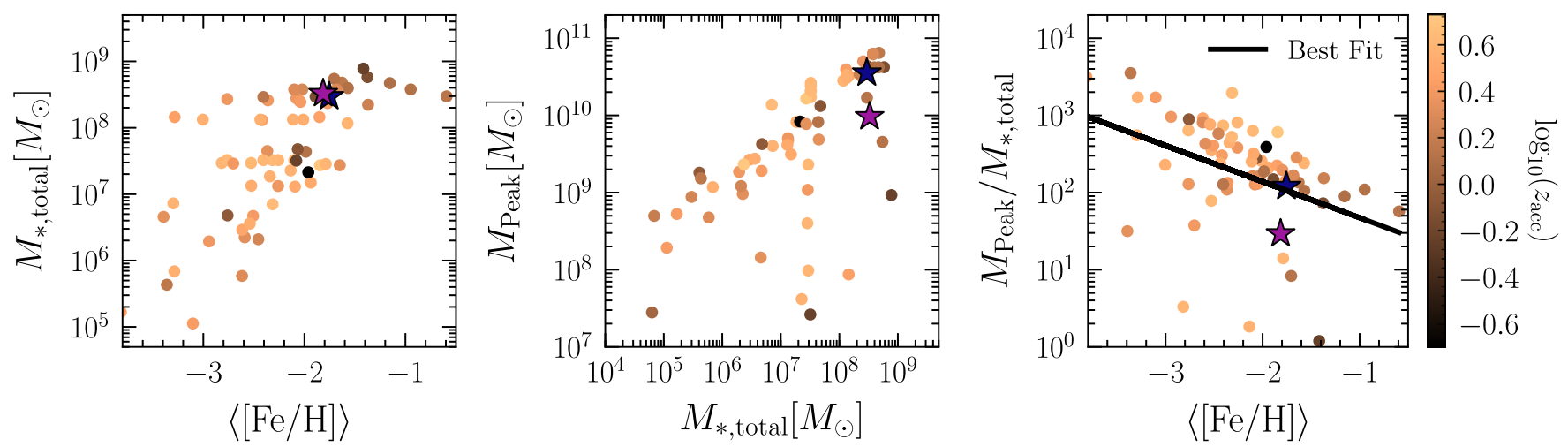

Figure 9. (Left) The relation of stellar mass and metallicity for the subhalos in $\mathrm{m} 12 \mathrm{i}$ that contribute stars within the solar circle. (Middle) The relation of peak halo mass and stellar mass for the same subhalos. (Right) The ratio of peak halo mass to stellar mass as a function of the average metallicity of each subhalo. The best-fit line, defined in Equation (4), is shown in solid black. In each panel, the stars correspond to Mergers I-II; their color convention matches that of Figure 1. The color of the points corresponds to the average accretion redshift for the stars in the merger.

panel of Figure 8 and reproduces the total DM distribution, as desired.

In reality, we do not know the exact DM mass fraction of each component, so we need a way to infer its value. To do so, it will be useful to recast Equation (2) as follows:

$$
f_{\mathrm{dm}}(v)=N\left(\xi_{*, \mathrm{r}} f_{\mathrm{r}}(v)+\frac{c_{\mathrm{I}}}{c_{\mathrm{r}}} \xi_{*, \mathrm{I}} f_{\mathrm{I}}(v)+\frac{c_{\mathrm{II}}}{c_{\mathrm{r}}} \xi_{*, \mathrm{II}} f_{\mathrm{II}}(v)\right),
$$

where $N$ is a normalization constant, and $c=M_{\mathrm{DM}} / M_{*}$ for each population. The value of $c$ tells us about the relative amount of DM and stars that each merger leaves at the solar circle. The DM-stellar mass fractions are provided in the third row of Table 2 , and the true values of $c_{\mathrm{I}(\mathrm{III})} / c_{\mathrm{r}}$ are provided in the sixth row.

To approximate the value of $M_{\mathrm{DM}} / M_{*}$ for a given merger, we will use its mass-to-light ratio. That is, we will assume that $c \approx M_{\text {peak }} / M_{*, \text { total }}$. Note that the relaxed population is itself the sum of several mergers. Moving forward, we treat these old mergers as a single population with some average metallicity and $M_{\text {peak }} / M_{*, \text { total }}$.
At first glance, this may seem like a poor approximation as the true $M_{\text {peak }} / M_{* \text {,total }}$ ratio (fourth row of Table 2) is larger than the corresponding $M_{\mathrm{DM}} / M_{*}$ ratio. However, the reduction between the two ratios is roughly consistent between the separate populations and thus cancels out when taking $c_{\mathrm{I}(\mathrm{II})} / c_{\mathrm{r}}$. We therefore conclude that $c \approx M_{\text {peak }} / M_{* \text {,total }}$ is an adequate approximation so long as the satellites lose roughly the same fraction of DM from their halos before reaching the solar circle.

To extrapolate the mass-to-light ratio, we use the present-day stellar mass-metallicity $\left(M_{*, \text { total }}-\langle[\mathrm{Fe} / \mathrm{H}]\rangle\right)$ and peak halo mass-stellar mass $\left(M_{\text {peak }}-M_{*, \text { total }}\right)$ relations. We now demonstrate this within the context of $\mathrm{m} 12 i$, saving a discussion of the Milky Way application to Section 6. The left and middle panels of Figure 9 show the $M_{* \text { total }}-[\mathrm{Fe} / \mathrm{H}]$ and $M_{\text {peak }}-M_{* \text {,total }}$ relations for m12i. ${ }^{17}$ Taken together, these can be used to obtain the dependence of the $M_{\text {peak }} / M_{* \text {,total ratio }}$

\footnotetext{
${ }^{17}$ Note that Figure 9 only includes the progenitor subhalos that eventually contribute debris within the solar circle. However, the corresponding relations for the Milky Way are provided for all observed dwarf galaxies at redshift $z=0$.
} 


\section{Host Halo m12i, All Dark Matter Components}

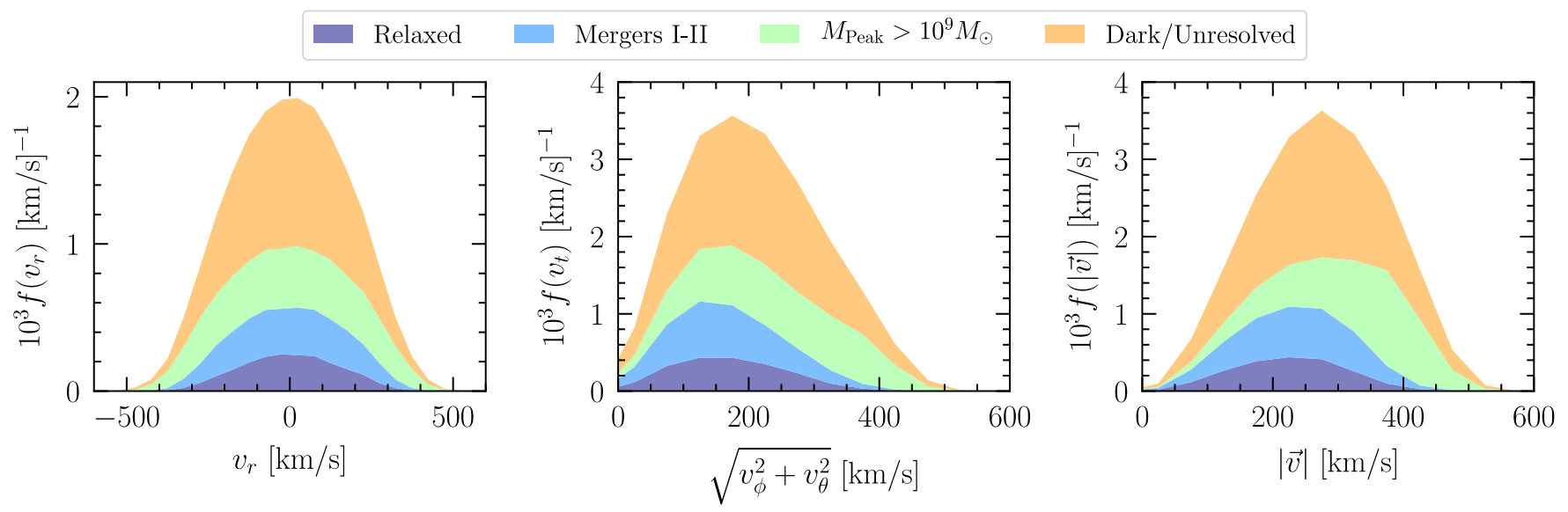

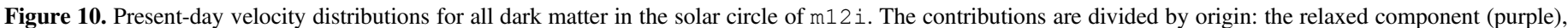

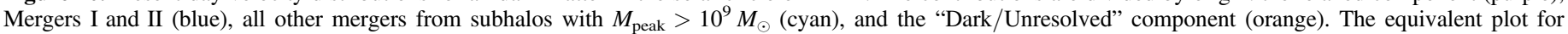
$\mathrm{m} 12 \mathrm{f}$ is provided as Figure 17 in the Appendix.

on $\langle[\mathrm{Fe} / \mathrm{H}]\rangle$, which is provided in the right panel of Figure 9. The mass-to-light ratio $M_{\text {peak }} / M_{* \text {,total }}$ is inversely proportional to the metallicity, with the more DM-dominated galaxies typically associated with more metal-poor stars. The approximately linear relationship is well fit by

$$
\log _{10}\left(\frac{M_{\text {peak }}}{M_{* \text {,total }}}\right)=1.20-0.47\langle[\mathrm{Fe} / \mathrm{H}]\rangle,
$$

indicated by the solid black line in Figure 9 (right). ${ }^{18}$ Given the average metallicities for Mergers I-II in $\mathrm{m} 12 i$, we infer that $M_{\text {peak }} / M_{*, \text { total }}=\{106,113\}$, respectively, compared to the true values $\{120,30\}$. The estimate for Merger II is a factor of $\sim 4$ too large, as can be seen from Figure 9 (right), where Mergers I-II are denoted by the colored stars. Such deviations can be properly accounted for by translating the spread in the fit to an error bar on the inferred $M_{\text {peak }} / M_{* \text {,total }}$; however, we ignore this for this simple illustrative example here. (We do a proper accounting of errors when applying this methodology to the Milky Way in the next section.) Similarly, we estimate that the relaxed population ${ }^{19}$ is comprised of mergers with $\left\langle M_{\text {peak }} / M_{* \text {,total }}\right\rangle=159$ given that their average metallicity is $\langle[\mathrm{Fe} / \mathrm{H}]\rangle=-2.13$.

Given an inferred $M_{\text {peak }} / M_{* \text {,total }}$ for each stellar component, we can estimate $c_{i} / c_{r}(i=\mathrm{I}$, II). The values for Mergers I-II of m12i are provided in the seventh row of Table 2, and they are within an order of magnitude of the true values. Using these weights in Equation (3), the distribution inferred from the stars is a good approximation of the underlying DM distribution, even if not an exact reproduction. The final result is shown in the right panel of Figure 8.

We apply the same procedure to $\mathrm{m} 12 \mathrm{f}$ and provide the corresponding figure in the Appendix as Figure 16. In this case,

\footnotetext{
18 The use of linear regression here is purely illustrative of the procedure, and more sophisticated fitting algorithms will be used in the next section when addressing the Milky Way.

${ }^{19}$ There are many ways to compute the mean of $M_{\text {peak }} / M_{* \text {,total }}$ of the relaxed population. In Table 2 , we present the values of the mean over all relaxed subhalos; however, these values might be artificially high. If one were to weigh the average by the subhalo mass for example, the value for m12i (m12 $\mathrm{f}$ ) would drop to $680(260)$.
}

the inferred values of $c_{i} / c_{r}$ are close to their true values (see Table 2) but the stellar distributions do not do a good job reconstructing the total DM. The failure is due to the discrepancy in the DM and stellar speed distribution for Merger I (a stream), which we discussed in Section 4.2.

\subsection{Untracked Component}

Next, we consider the DM in the "Dark/Unresolved" component. As already discussed, this component consists of DM that originates from subhalos whose galaxies are not adequately resolved, truly dark subhalos, unresolved subhalos, or smooth accretion. In the first case, the component may actually be tracked by stars. For the other cases, we do not expect stars to be brought in along with the DM. Because we cannot further distinguish between these separate contributions, we conservatively group them together and study their total velocity distribution.

Figure 10 plots the radial, tangential, and speed distributions for the "Dark/Unresolved" component of m12i. The distributions are stacked on top of the distributions for the relaxed population and Mergers I-II. We also include the contribution from DM that originates from subdominant mergers with $M_{\text {peak }}>10^{9} M_{\odot}$; this contribution is similar to that of Mergers I-II. The additional DM from the "Dark/Unresolved" component has two important effects. First, it decreases the overall dispersion in the radial velocity, smoothening out the kinematic structure left behind by the recent mergers. Second, it shifts the peak in the speed distribution to a value that lies closer to (but still above) that of the relaxed component. As we see from Figure 1, the "Dark/Unresolved" contribution enters the solar circle at redshift $z_{\text {acc }} \lesssim 2$, which explains why its overall speed is faster, on average, than that of the relaxed component.

We emphasize that it is not possible to infer the fraction of DM originating from smooth accretion and/or dark subhalos in the Milky Way directly from simulations. The primary challenge is that both depend sensitively on the accretion history of the simulated host halo, which may not replicate that of the Milky Way. The wide halo-to-halo variation has already been underscored by a separate study of 10 Aquarius halos (Wang et al. 2011), which found large variations in the fractional contribution of each population between different 
Milky Way realizations. It is therefore imperative to develop methods to characterize the DM contribution from smooth accretion and dark subhalos empirically. This requires its own dedicated study.

\section{The Local Dark Matter in the Milky Way from Luminous Satellites}

We now apply the formalism developed in Sections 4 and 5 to our own Galaxy with the aim of empirically inferring the local speed distribution for DM sourced from luminous satellites. Necib et al. (2019) characterized the velocity distribution of the local accreted stellar population using a cross-match of Gaia DR2 data (Lindegren et al. 2016; Gaia Collaboration et al. 2018) and SDSS (Ahn et al. 2012). They characterized a metal-poor "halo" population with average metallicity $\langle[\mathrm{Fe} / \mathrm{H}]\rangle_{\text {halo }}=-1.82$ that is nearly isotropic and comprises $\sim 24 \%$ of the local accreted stars within heliocentric distances of $4 \mathrm{kpc}$ and above $|z|>2.5 \mathrm{kpc}$ of the midplane. ${ }^{20} \mathrm{It}$ is the parallel of the relaxed population discussed in Section 4.1. The Milky Way's relaxed component constitutes a larger fraction of the stellar halo and is moderately more metal-rich than that of $m 12 i$ or m12f.

Additionally, the authors characterized the kinematics of a younger stellar population with average metallicity $\langle[\mathrm{Fe} / \mathrm{H}]\rangle_{\text {subs }}=$ -1.39 . This substructure, referred to as the Gaia Sausage or Gaia Enceladus, is an example of debris flow. Like Merger I of m12i, its velocity distribution is highly radial and spatially uniform within the SDSS footprint. However, it contributes a much larger fraction of the local accreted stars $(\sim 76 \%)$ than does Merger I of m12i ( 40\%).

As the inner Milky Way appears to be dominated by the stellar debris of one single large merger, its composition is simpler than that of either m12i or m12f. Consequently, we need only consider the sum of two terms when building the distribution of local DM speeds in the Galaxy:

$$
f_{\mathrm{dm}}(v)=N\left(\xi_{* \text {,halo }} f_{\text {halo }}(v)+\frac{c_{\text {subs }}}{c_{\text {halo }}} \xi_{* \text {,subs }} f_{\text {subs }}(v)\right),
$$

where the first term corresponds to the relaxed component and the second term corresponds to the substructure. Note that we identify these contributions with the terms "halo" and "subs" as in Necib et al. (2019). The ratio $c_{\text {subs }} / c_{\text {halo }}$ can be determined following the procedure outlined in Section 5.1, but using relations specific to the Milky Way.

We adopt the $M_{*, \text { total }}-[\mathrm{Fe} / \mathrm{H}]$ relation from Kirby et al. (2013):

$$
\begin{aligned}
\langle[\mathrm{Fe} / \mathrm{H}]\rangle= & (-1.69 \pm 0.04) \\
& +(0.30 \pm 0.02) \log _{10}\left(\frac{M_{*, \text { total }}}{10^{6} M_{\odot}}\right),
\end{aligned}
$$

which applies to dwarf galaxies of the Milky Way at redshift $z=0$. The root mean square about the best-fit line is 0.17 dex. This linear relation holds over many orders of magnitude in stellar mass, from $M_{* \text {,total }} \sim 10^{4}-10^{9} M_{\odot}$. Data from SDSS suggest that the trend roughly continues up to $M_{* \text {,total }} \sim 10^{12} M_{\odot}$ (Gallazzi et al. 2005). Equation (6) is similar to the $M_{* \text {,total }}-[\mathrm{Fe} / \mathrm{H}]$ relation recovered in the FIRE-2 simulations (see e.g., Figure 9).

\footnotetext{
${ }^{20}$ Note that the volume of study in Necib et al. (2019) is outside the solar circle, as defined in this work.
}

However, while the simulations reproduce the observed slope, they find systematically lower values of iron abundance (Escala et al. 2018). This offset is likely due to specific choices made in the modeling of the delay time distribution and yields of Type Ia supernovae.

The Kirby et al. (2013) relation applies to observed dwarf galaxies at redshift $z=0$, while the desired quantity is the stellar mass of galaxies disrupted at earlier redshifts. In this work, we assume that there is no redshift dependence to the stellar mass-metallicity relation. To estimate the size of this dependence, we can combine Equation (6) with the redshift evolution inferred from the simulations. Taking as an example the work of Ma et al. (2016), we assume a shift in average metallicity that goes as $\Delta[\mathrm{Fe} / \mathrm{H}]=0.67[(\exp (-0.5 z)-1]$. For a merger at redshift $z=1$, this leads to $\Delta[\mathrm{Fe} / \mathrm{H}]=$ -0.26 . A merger at redshift $z=3$ is associated with a shift of -0.52 . This correction shifts the expected metallicity down by some constant at any given redshift. In our case, though, we are only interested in the relative difference in metallicities between the substructure and halo populations, and this does not change with redshift evolution. As a result, $c_{\text {subs }} / c_{\text {halo }}$ is unaffected.

To estimate the peak halo mass, we follow the same procedure outlined by Garrison-Kimmel et al. (2017a). Above $M_{\text {peak }} \gtrsim 10^{11.5} M_{\odot}$, this $M_{\text {peak }}-M_{* \text {,total }}$ relation maps onto that of Behroozi et al. (2013a), which has a constant log-normal scatter of $\sigma=0.2$ dex about the median value of $M_{* \text {,total }}$. For lower-mass galaxies with $M_{\text {peak }} \lesssim 10^{11.5} M_{\odot}$, the stellar mass is effectively a power law in peak halo mass. Specifically, $M_{*} \propto M_{\text {peak }}^{\alpha}$ where the slope $\alpha$ depends on the assumed lognormal scatter, $\sigma_{v}$, about the mean value of $M_{* \text {,total }}$. We use the growing-scatter model of Garrison-Kimmel et al. (2017a), where the value of $\sigma_{v}$ is allowed to grow linearly as $\log _{10} M_{\text {peak }}$ decreases. That is,

$$
\sigma_{v}=0.2+v \times\left(\log _{10} M_{\text {peak }}-\log _{10} M_{1}\right),
$$

where $M_{1} \sim 10^{11.5} M_{\odot}$ and $v$ sets how the scatter increases. The best-fit power-law slope in this case is

$$
\alpha \simeq 0.25 v^{2}-1.37 v+1.69 .
$$

We take $v=-0.1$ as our benchmark value.

We note that this $M_{*, \text { total }}-M_{\text {peak }}$ relation was derived for DM-only simulations and that the presence of a baryonic disk can have important effects. The expectation is that the disk will tidally destroy infalling subhalos, requiring that the predicted $M_{* \text {,total }}$ (for given $M_{\text {peak }}$ ) must be shifted to higher values in order to recover the Milky Way's cumulative stellar mass function (Garrison-Kimmel et al. 2017b). This, in turn, would result in a more shallow power-law falloff.

We perform a Monte Carlo procedure to estimate the relative amount of local DM in the substructure as opposed to the halo population (e.g., $c_{\text {subs }} / c_{\text {halo }}$ ). The procedure is as follows:

1 . We use the $M_{\text {peak }}-M_{* \text {,total }}$ relation to estimate the associated stellar mass, for a given $M_{\text {peak }}$. The value of $M_{* \text {,total }}$ is randomly selected from a normal distribution with the mean given by the growing-scatter model of Garrison-Kimmel et al. (2017a), with self-consistent $v$, $\sigma_{v}$, and $\alpha$ from Equation (7) and Equation (8). This yields a prediction for the $M_{\text {peak }} / M_{* \text {,total }}$ ratio. We demand that $M_{\text {peak }}>5 M_{*, \text { total }}$. 


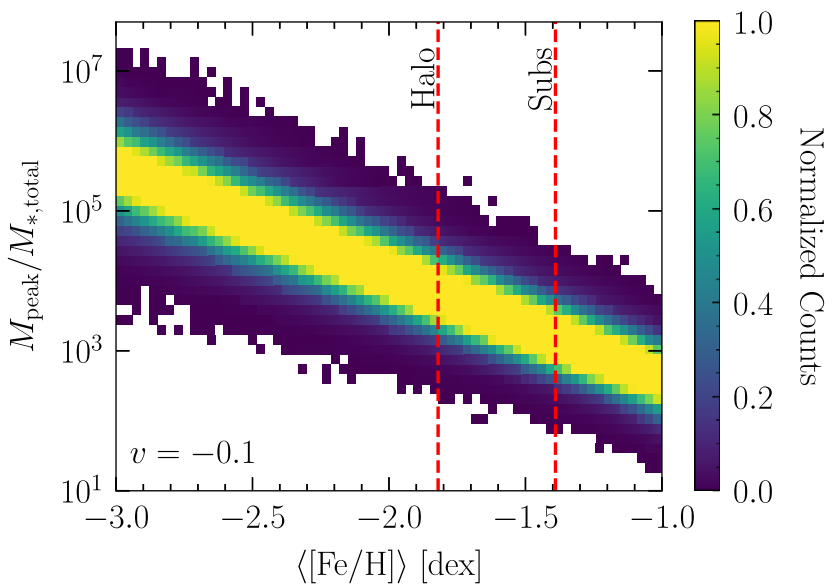

Figure 11. Estimated $M_{\text {peak }} / M_{* \text {,total }}-\langle[\mathrm{Fe} / \mathrm{H}]\rangle$ relation, assuming the growing-scatter model of Garrison-Kimmel et al. (2017a) with $v=-0.1$. The average metallicities of the halo and substructure components in the Milky Way, as derived in Necib et al. (2019), are indicated by the red dashed lines.

2. Using this stellar mass, we estimate the metallicity by randomly selecting $\langle[\mathrm{Fe} / \mathrm{H}]\rangle$ from a normal distribution with mean given by Equation (6) and dispersion of $\sim 0.17$ dex.

3. We repeat the previous two steps 500 times to build a distribution of $M_{\text {peak }} / M_{* \text {,total }}$ versus $\langle[\mathrm{Fe} / \mathrm{H}]\rangle$. The result is shown in Figure 11.

4. We randomly select a point with metallicity $\langle[\mathrm{Fe} / \mathrm{H}]\rangle \sim-1.39$, as per the substructure population, and another with metallicity $\langle[\mathrm{Fe} / \mathrm{H}]\rangle \sim-1.82$, as per the halo population. The ratio of their respective $M_{\text {peak }} / M_{* \text {,total }}$ values yields the $c_{\text {subs }} / c_{\text {halo }}$ weighting factor. Repeating this $8 \times 10^{6}$ times allows us to quantify the 16th-50th-84th percentiles of this factor.

For the $v=-0.1$ benchmark, we find that

$$
\frac{c_{\text {subs }}}{c_{\text {halo }}}=0.23_{-0.15}^{+0.43} \text {. }
$$

As a point of comparison, we also obtain the ratios for constant $\sigma_{v}$. For $\sigma_{v}=0.3(2.0)$, Equation (9) yields $c_{\text {subs }} / c_{\text {halo }}=$ $0.22_{-0.14}^{+0.38}\left(0.15_{-0.14}^{+2.60}\right)$. Substituting the value of Equation (9) back into Equation (5), we find that $42_{-22}^{+26} \%$ of the local DM that originates from luminous satellites is in the debris flow. ${ }^{21}$ This value is consistent, within the range of uncertainty, with values estimated using kinematic arguments in Evans et al. (2018), but is larger than the value found from estimates with the Auriga simulations in Fattahi et al. (2019). We, however, emphasize that this quoted fraction is taken with respect to the luminous merging satellites alone, and therefore the fraction of DM in the Gaia Sausage with respect to all DM is smaller than $42_{-22}^{+26} \%$.

One might notice that Equation (9) is systematically lower than the reweighting factors found for $\mathrm{m} 12 i$. This is because there is a greater difference in metallicity between the relaxed and substructure populations in m12 $i$, compared to what is observed in the Milky Way. Because the halo and substructure

\footnotetext{
${ }^{21}$ To simplify this calculation, we did not convolve the error on the stellar fraction from the best fit in Necib et al. (2019). We expect it to be subdominant to the error from Equation (9).
}

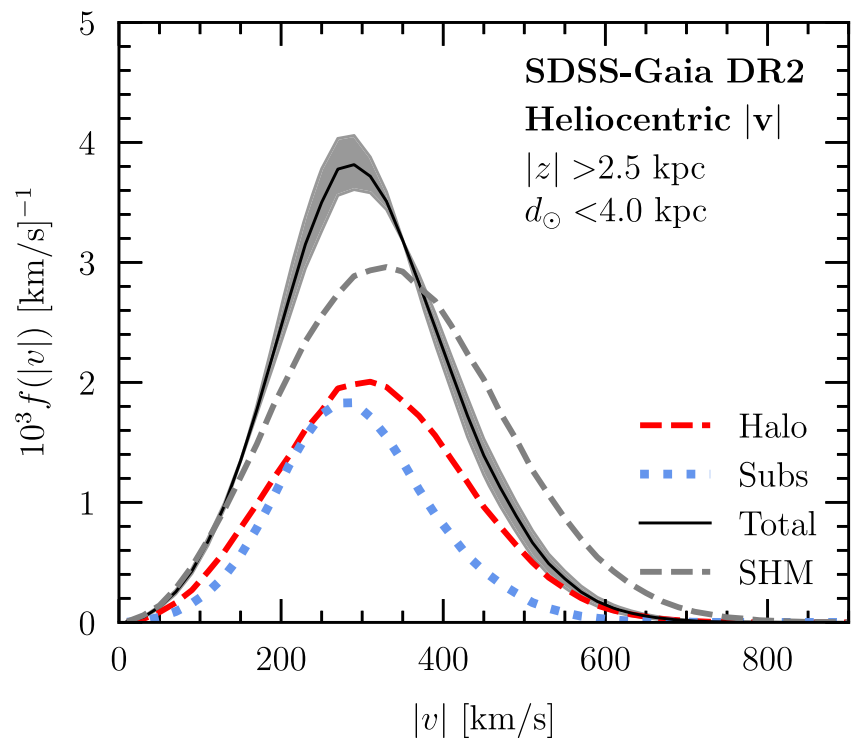

Figure 12. Updated heliocentric velocity distribution from Necib et al. (2019). This distribution takes into account the relative dark matter contribution between the substructure and the halo component, as given by Equation (9). This distribution applies to the dark matter accreted from luminous satellites and does not account for potential contributions from dark subhalos or smooth accretion. Data for this distribution are publicly available at https://linoush. github. io/DM_Velocity_Distribution/.

populations in the Milky Way are closer to each other in average metallicity, the amount of DM that each contributes is commensurate between the two.

Figure 12 shows the heliocentric velocity distribution of DM sourced from luminous satellites, as inferred from the SDSSGaia DR2 data. The halo and substructure distributions (red dashed and blue dotted, respectively) were derived in Necib et al. (2019). When summing their contributions (black solid), the relative fraction is set by Equations (5) and (9). The gray band denotes the uncertainty from the inferred value of $c_{\text {subs }} / c_{\text {halo }}$. For comparison, we also show the Standard Halo Model (gray dashed), assuming a Maxwell-Boltzmann distribution with a dispersion $\sigma=220 / \sqrt{2} \mathrm{~km} \mathrm{~s}^{-1}$.

\section{Conclusions}

In this paper, we studied two cosmological zoom-in hydrodynamic simulations of Milky-Way-mass galaxies from the Latte suite of the FIRE-2 simulations. Our primary goal was to understand how the DM and stars accreted from luminous satellite galaxies trace each other in the inner regions of a Milky-Way-like galaxy. In each of these host galaxies, we focused on the accreted material in the solar circle (defined as $\left|r-r_{\odot}\right|<2 \mathrm{kpc}$ and $|z| \leqslant 1.5 \mathrm{kpc}$ with $r_{\odot}$ the solar radius), which is most relevant for ground-based DM direct detection experiments.

The accreted DM and stars in the solar circle can be divided into three separate components whose general behavior is summarized as follows:

1. The "relaxed" DM and stellar component is accreted from the oldest mergers $\left(z_{\text {acc }} \gtrsim 3\right)$. At these early times, the protogalaxy is still evolving, and changes to the galactic potential redistribute the energies of the DM and stellar debris, mixing them fully in phase space. As 
a result, the present-day velocity distributions of the DM and stars from these oldest mergers are well correlated. The metal-poor subcomponent of local stars is an adequate proxy for the relaxed population. We find that stars with metallicity $[\mathrm{Fe} / \mathrm{H}] \lesssim-2$ to -3 trace the relaxed distributions reasonably well in the Latte hosts, consistent with previous results from Herzog-Arbeitman et al. (2018a). It is possible that the low-metallicity sample may be contaminated by stars from more recent mergers whose metallicity distributions have lowmetallicity tails. Statistical clustering algorithms-such as that used in Necib et al. (2019)_can ameliorate such contamination.

2. Once the protogalaxy is in place, smaller mergers continue to the present day. The tidal debris from these mergers evolves in phase space following Liouville's theorem. As a satellite falls into the galaxy, it leaves behind a trail of tidal debris. If the time since infall is relatively short, then this material is typically in a stream and is clustered in both position and velocity space. In such cases, we find that there can be significant spatial variations in the DM and stars, which lead to discrepancies in their velocity distributions.

3 . If the time since infall is longer and the satellite has completed multiple orbital wraps, then the spatial distribution of its tidal debris is well mixed, but the kinematic substructure is still preserved. This class of substructure is referred to as debris flow. We find that the velocity and spatial distributions of the DM and stars from these mergers are well correlated. Unlike the case of stellar streams, the distributions do not exhibit large local variations.

As we have demonstrated with the Latte FIRE-2 simulations, the DM-stellar correlations are robust for both the relaxed and debris flow populations, and hold despite the significant differences in the merger histories of the two host halos studied here. The conclusions are specific to the solar circle, where our study is focused. For the most significant mergers (Mergers I-IV in Table 1), we find that much of the halo has been stripped off by the time the satellite has sunk to the solar radius. As a result, the DM being removed as the satellite passes through the solar circle is the most bound, similar to the stars.

In the case of streams, care needs to be taken in extrapolating the kinematic DM properties from the stellar distributions due to large localized variations that can arise. Dedicated simulations may be needed to better quantify the expected discrepancies between the DM and stellar debris from a particular merger. Such simulations may be warranted to study the potential DM contribution from stellar streams, such as S1, in the solar neighborhood (Myeong et al. 2018a, 2018c; O'Hare et al. 2018).

The total DM velocity distribution at the solar circle can be built up from the separate components described above if one can infer the relative amounts of DM brought in by each merger. We provide a simple procedure to do so, which combines the mass-metallicity relation with abundance matching to relate the $M_{\text {peak }} / M_{* \text {,total }}$ ratio to the average metallicity $\langle[\mathrm{Fe} / \mathrm{H}]\rangle$ of a merger. This relation allows us to estimate the relative amounts of DM to stars brought in by each merger. In this way, we can build the total velocity distribution for the DM associated with luminous mergers.
The results of our work on the Latte hosts is pertinent in light of the recently discovered stellar debris field in the solar neighborhood (Belokurov et al. 2018; Helmi et al. 2018). These stars can be divided into a metal-poor and nearly isotropic population and a more metal-rich and radially biased population. Using a Gaussian clustering algorithm, Necib et al. (2019) recently extracted the velocity distributions of these two components using data from the SDSS-Gaia cross-match. The two components correspond to a relaxed stellar population and debris flow and should be well traced by the DM removed from the same set of mergers, following our study of the Latte hosts. Using the rescaling relations from Section 6, we estimate that $42_{-22}^{+26} \%$ of the local DM accreted from luminous satellites is in debris flow.

The method described in this paper does not, by assumption, account for DM contributions from dark subhalos or smooth accretion, which should not be associated with stars. In the Latte hosts studied here, we are not able to distinguish this contribution from DM arising from unresolved DM (sub)halos or halos whose galaxies are not resolved, which can contribute up to $50 \%$ of the local DM fraction. However, the distinguishing power will improve as the stellar and DM mass resolution improves. In the Latte hosts, this DM contribution (which we label as "Dark/Unresolved") comes in at redshifts $z \lesssim 2$, so it has, on average, larger speeds than the older relaxed component.

It is challenging to extract conclusions regarding dark subhalos or smooth accretion in simulations of our own Galaxy. Previous studies using high-resolution DM-only $\mathrm{N}$-body simulations have found considerable variation in the potential origin of DM in the solar neighborhood. For example, the DM halo in the Via Lactea simulation is rapidly built up around redshift $z \sim 1.7$ and then remains essentially stationary until present time (Diemand et al. 2007). In some Aquarius halos, the DM in the solar neighborhood is nearly all in place before $z \sim 6$, whereas in others, most of the DM accreted more recently (Wang et al. 2011). This variation underscores the importance of studying a variety of simulated halos to better understand how the fraction of local DM from dark subhalos or smooth accretion depends on merger history. Only in this way can we robustly extrapolate conclusions to the Milky Way.

Finally, we emphasize that all results regarding the DMstellar correspondence that we draw from the FIRE-2 simulations assume cold, collisionless DM. It will be important to understand how these conclusions generalize to a broader class of DM models where the DM and stellar trajectories may be different, by assumption. Some classic examples include selfinteracting or ultralight scalar DM models.

For readers who would like to use the empirical velocity distributions from Necib et al. (2019) to model the local DM distribution from luminous satellites, we provide interpolated functions at https://linoush.github.io/DM_Velocity_ Distribution/. The separate contributions from the halo and substructure distributions can be combined following the prescription in Section 6.

We thank V. Belokurov, E. Kirby, A. Peter, and D. Spergel for useful conversations.

L.N. is supported by the DOE under award number DESC0011632, and the Sherman Fairchild fellowship. M.L. is supported by the DOE under award number DESC0007968 and the Cottrell Scholar Program through the Research 
Corporation for Science Advancement. Support for S.G.K. was provided by NASA through Einstein Postdoctoral Fellowship grant No. PF5-160136 awarded by the Chandra X-ray Center, which is operated by the Smithsonian Astrophysical Observatory for NASA under contract NAS8-03060. A.W. was supported by NASA through ATP grant 80NSSC18K1097 and grants HST-GO-14734 and HST-AR-15057 from STScI. C.A.F.G. was supported by NSF through grants AST-1517491, AST-1715216, and CAREER award AST-1652522, by NASA through grants NNX15AB22G and 17-ATP17-0067, and by a Cottrell Scholar Award from the Research Corporation for Science Advancement. Support for P.F.H., S.G.K., and R.E.S. was provided by an Alfred P. Sloan Research Fellowship, NSF Collaborative Research Grant \#1715847 and CAREER grant \#1455342, and NASA grants NNX15AT06G, JPL 1589742, and 17-ATP17-0214.

Numerical calculations were run on the Caltech compute cluster "Wheeler," allocations from XSEDE TG-AST130039 and PRAC NSF.1713353 supported by the NSF, and NASA HEC SMD-16-7592. D.K. was supported by NSF grant AST1715101 and the Cottrell Scholar Award from the Research
Corporation for Science Advancement. This work was performed in part at the Aspen Center for Physics, which is supported by National Science Foundation grant PHY1607611. We used computational resources from the Extreme Science and Engineering Discovery Environment (XSEDE), supported by NSF.

Software: Astropy (Astropy Collaboration et al. 2013), IPython (Pérez \& Granger 2007), GIZMO (Hopkins 2015), GADGET-3 (Springel 2005), STARBURST99 v7.0 (Leitherer et al. 1999, 2014), Rockstar (Behroozi et al. 2013b).

\section{Appendix}

In this appendix, we provide some additional figures that supplement the discussion in the main text. Figure 13 compares the velocity distributions of the relaxed populations to those of stars with $[\mathrm{Fe} / \mathrm{H}]<-3$. Figures 14 and 15 show the velocity distributions of other significant mergers in $\mathrm{m} 12 i$ and $\mathrm{ml} 2 \mathrm{f}$. Figure 16 shows the results of estimating the total DM distribution in $\mathrm{m} 12 \mathrm{f}$, and Figure 17 plots the velocity distributions for all DM components in $\mathrm{m} 12 \mathrm{f}$.

FIRE Host Halo m12f, Relaxed Component
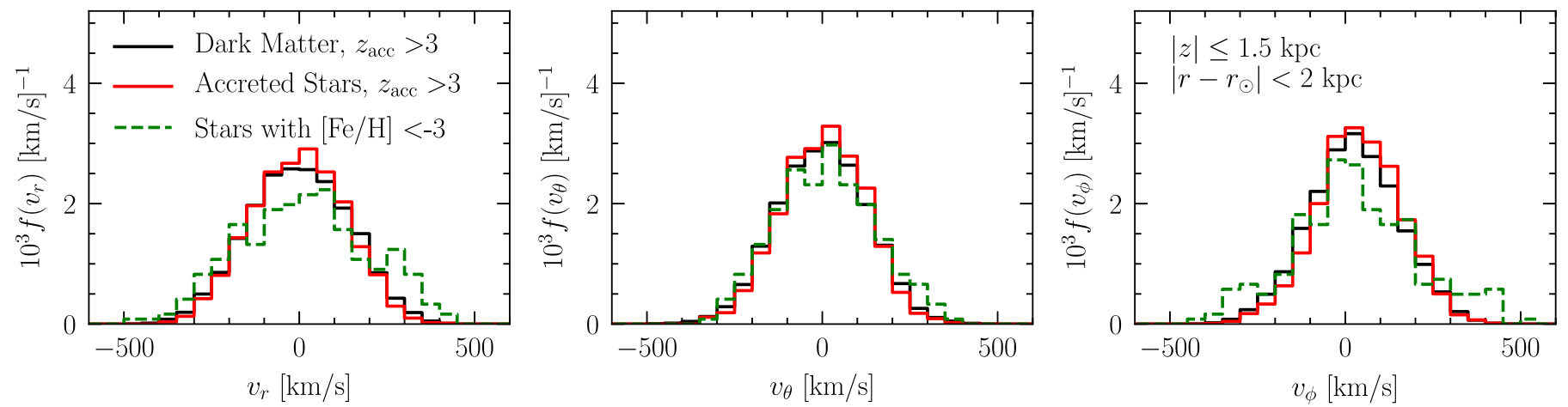

FIRE Host Halo m12i, Relaxed Component
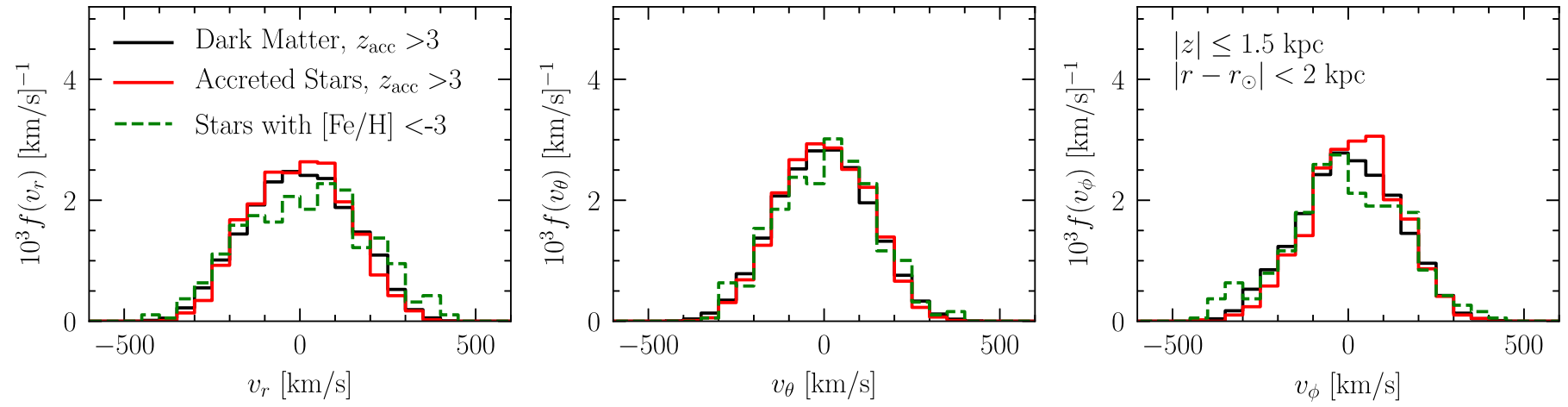

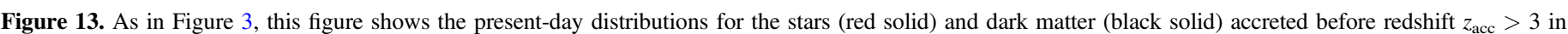

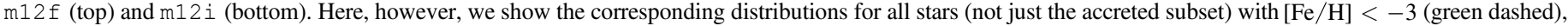
as opposed to $[\mathrm{Fe} / \mathrm{H}]<-2$. 


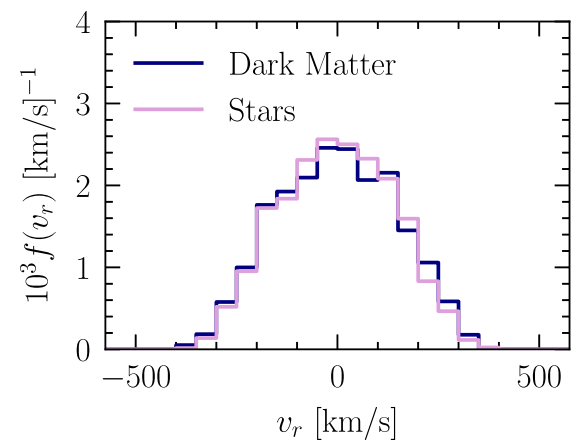

FIRE Host Halo m12i Merger II
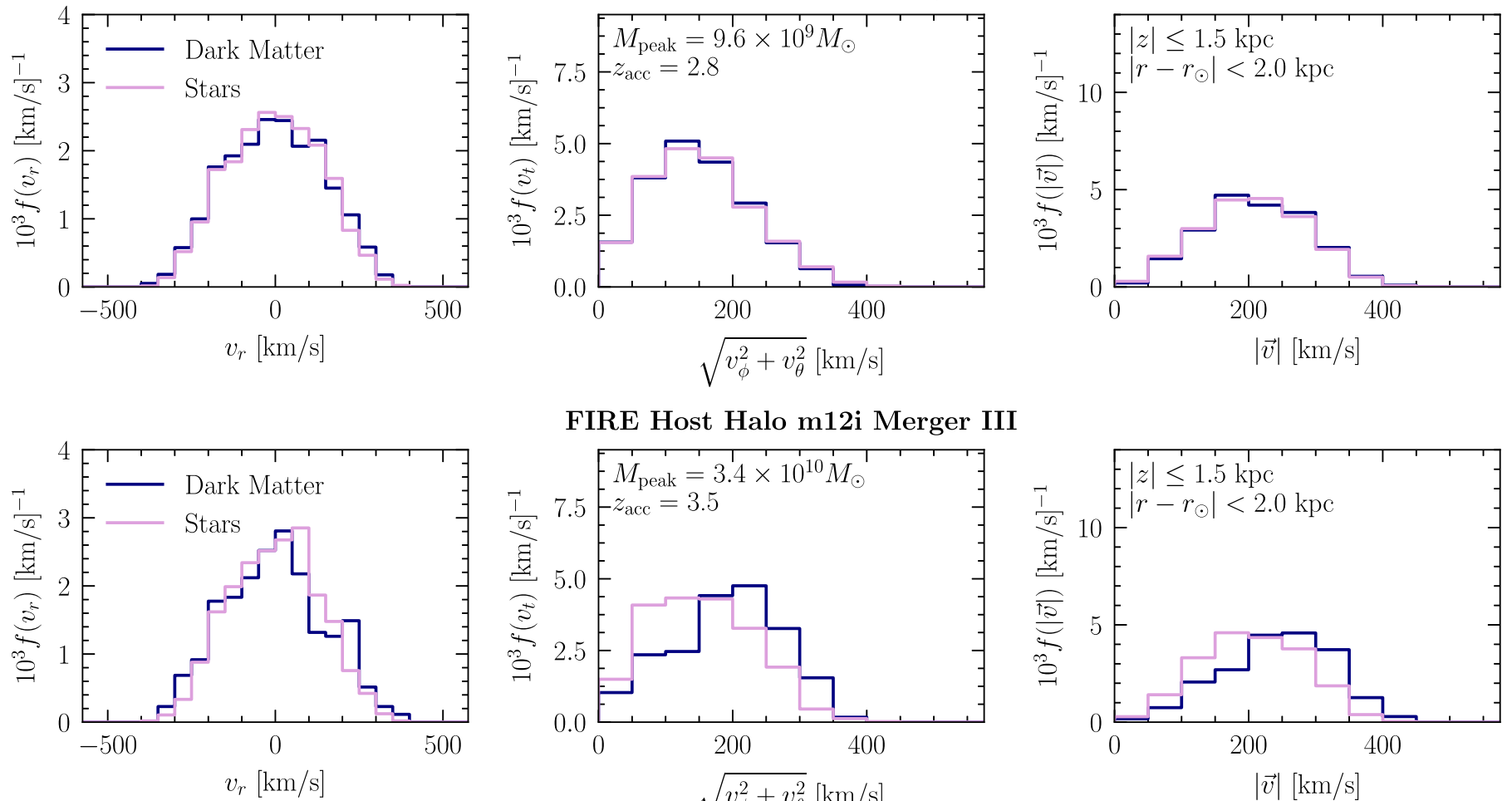

FIRE Host Halo m12i Merger III
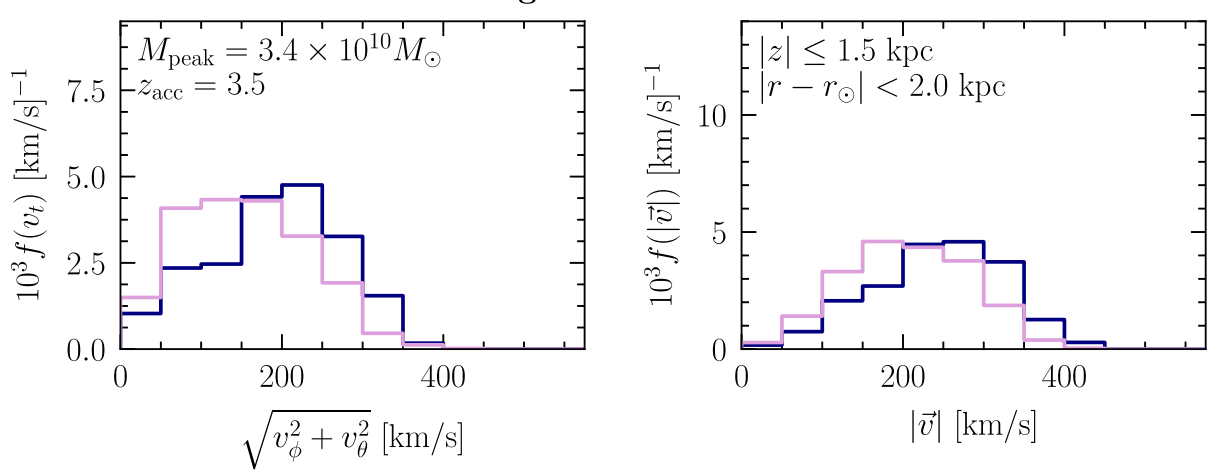

FIRE Host Halo m12i Merger IV
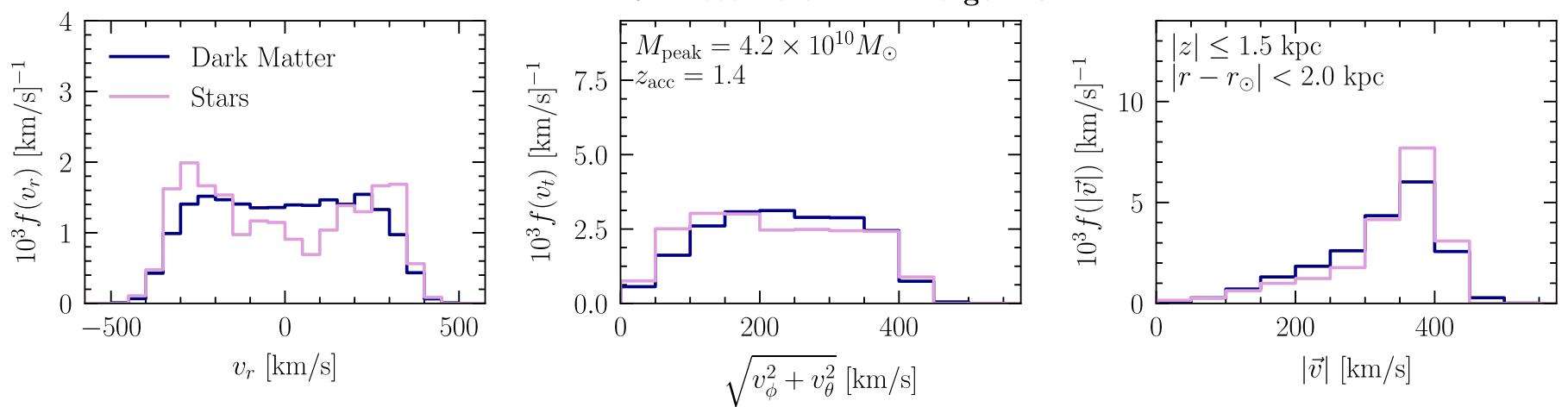

Figure 14. Same as Figure 5, except for Mergers II, III, and IV of m12i. 


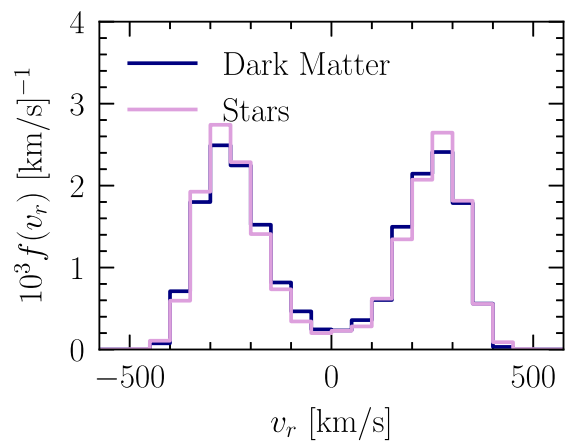

FIRE Host Halo m12f Merger II
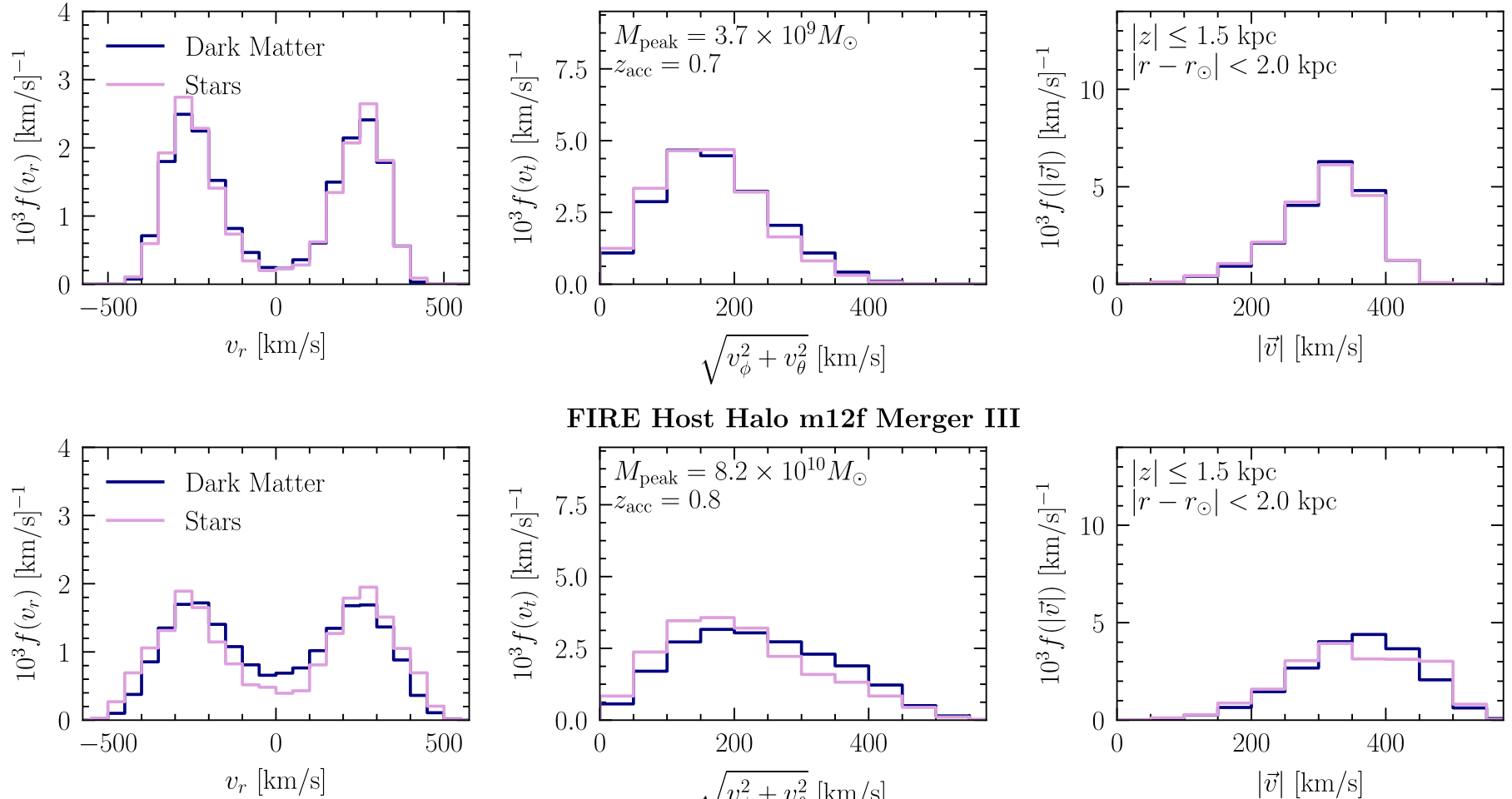

FIRE Host Halo m12f Merger III
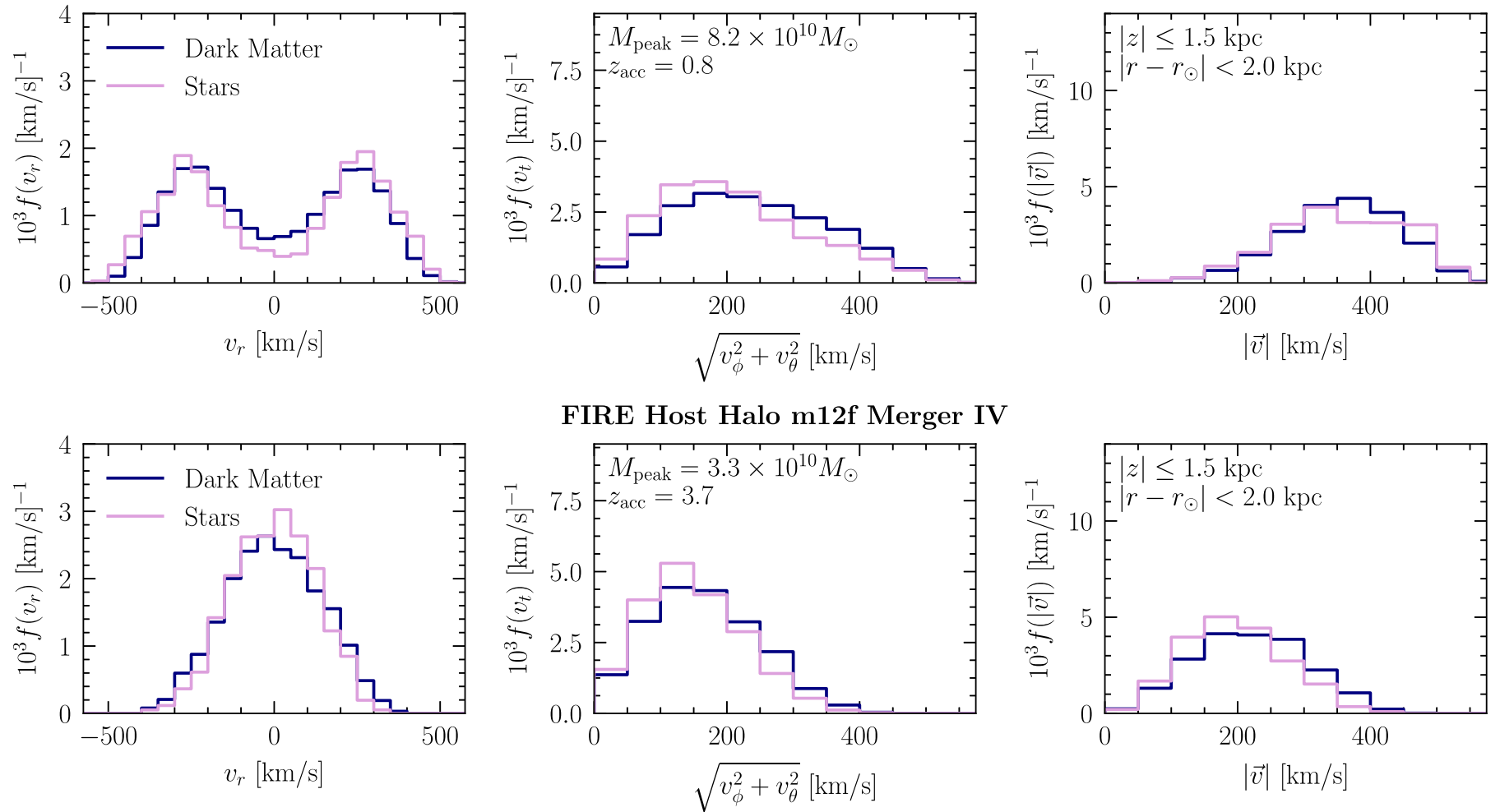

Figure 15. Same as Figure 5, except for Mergers II, III, and IV of m12f.
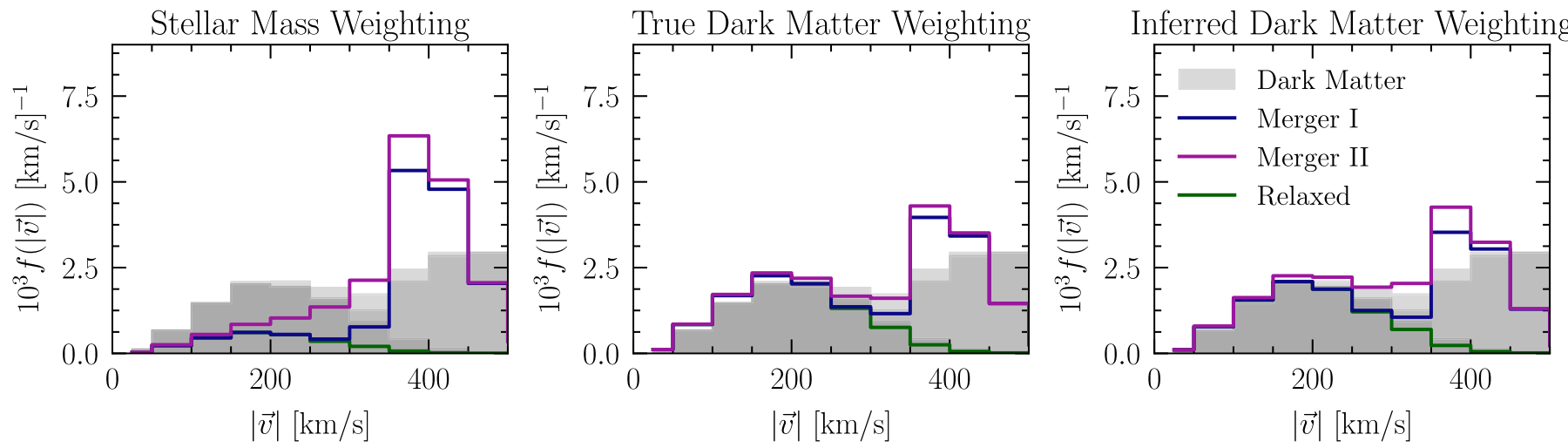

Figure 16. Same as Figure 9, except for $\mathrm{m} 12 \mathrm{f}$. 


\section{Host Halo m12f, All Dark Matter Components}

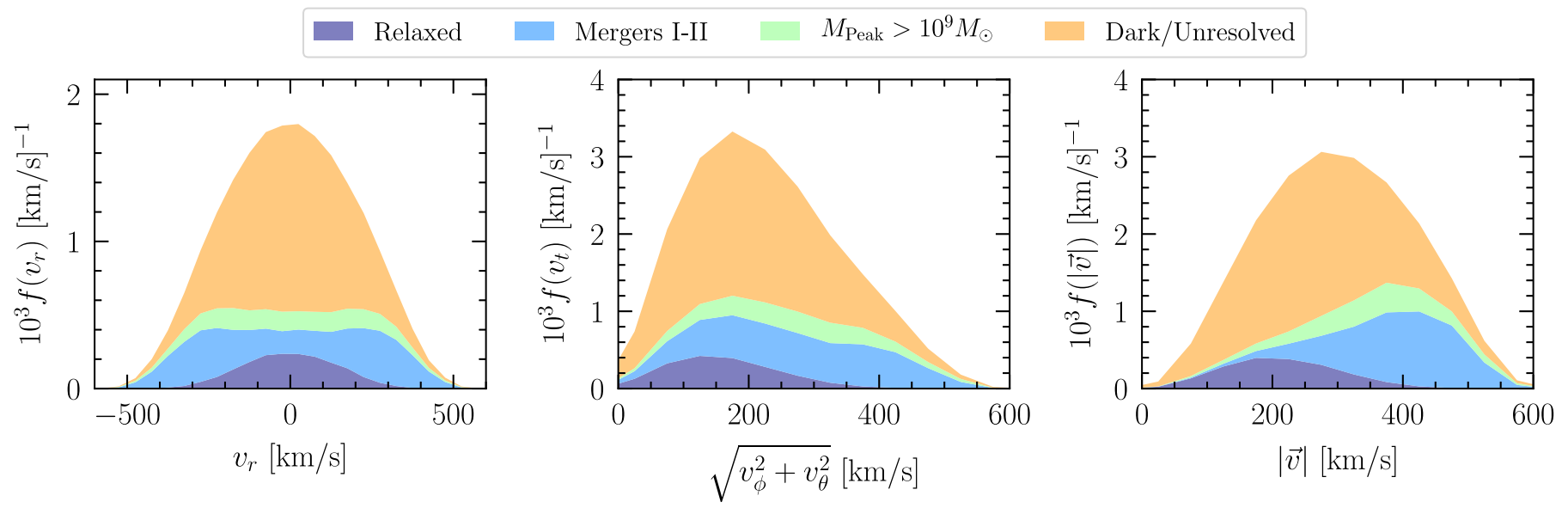

Figure 17. Same as Figure 10, except for m12f. Comparing Figures 10 and 17 to a Maxwell-Boltzmann distribution with dispersion $220 / \sqrt{2} \mathrm{~km} \mathrm{~s}^{-1}$, we find that the FIRE distributions are peaked at larger speeds of $\sim 275 \mathrm{~km} \mathrm{~s}^{-1}$ than the Standard Halo Model, which is peaked at $220 \mathrm{~km} \mathrm{~s}^{-1}$.

\section{ORCID iDs}

Lina Necib (ib https://orcid.org/0000-0003-2806-1414 Shea Garrison-Kimmel ib https://orcid.org/0000-00024655-8128

Andrew Wetzel (10) https://orcid.org/0000-0003-0603-8942

Robyn Sanderson (i) https://orcid.org/0000-0003-3939-3297

Philip F. Hopkins (D) https://orcid.org/0000-0003-3729-1684

Claude-André Faucher-Giguère (ib https://orcid.org/0000-

0002-4900-6628

Dušan Kereš (iD https://orcid.org/0000-0002-1666-7067

\section{References}

Ahn, C. P., Alexandroff, R., Allende Prieto, C., et al. 2012, ApJS, 203, 21 Astropy Collaboration, Robitaille, T. P., Tollerud, E. J., et al. 2013, A\&A, 558, A33

Behroozi, P. S., Wechsler, R. H., \& Conroy, C. 2013a, ApJ, 770, 57

Behroozi, P. S., Wechsler, R. H., \& Wu, H.-Y. 2013b, ApJ, 762, 109

Belokurov, V., Erkal, D., Evans, N. W., Koposov, S. E., \& Deason, A. J. 2018, MNRAS, 478, 611

Bland-Hawthorn, J., \& Gerhard, O. 2016, ARA\&A, 54, 529

Bonaca, A., Conroy, C., Wetzel, A., Hopkins, P. F., \& Keres, D. 2017, ApJ, 845,101

Breddels, M. A., \& Helmi, A. 2013, A\&A, 558, L3

Bullock, J. S., \& Johnston, K. V. 2005, ApJ, 635, 931

Bullock, J. S., Kravtsov, A. V., \& Weinberg, D. H. 2001, ApJ, 548, 33

Cooper, A. P., Parry, O. H., Lowing, B., Cole, S., \& Frenk, C. 2015, MNRAS, 454, 3185

De Lucia, G., \& Helmi, A. 2008, MNRAS, 391, 14

Deason, A. J., Belokurov, V., Koposov, S. E., \& Lancaster, L. 2018, ApJL, 862, L1

Deason, A. J., Mao, Y.-Y., \& Wechsler, R. H. 2016, ApJ, 821, 5

Diemand, J., Kuhlen, M., \& Madau, P. 2007, ApJ, 667, 859

Diemand, J., Kuhlen, M., Madau, P., et al. 2008, Natur, 454, 735

Elahi, P. J., Thacker, R. J., \& Widrow, L. M. 2011, MNRAS, 418, 320

Escala, I., Wetzel, A., Kirby, E. N., et al. 2018, MNRAS, 474, 2194

Evans, N. W., O'Hare, C. A. J., \& McCabe, C. 2018, arXiv:1810.11468

Fattahi, A., Belokurov, V., Deason, A. J., et al. 2019, MNRAS, 484, 4471

Faucher-Giguère, C.-A., Lidz, A., Zaldarriaga, M., \& Hernquist, L. 2009, ApJ, 703,1416

Font, A. S., Johnston, K. V., Bullock, J. S., \& Robertson, B. 2006, ApJ, 638,585

Font, A. S., McCarthy, I. G., Crain, R. A., et al. 2011, MNRAS, 416, 2802

Gaia Collaboration, Brown, A. G. A., Vallenari, A., et al. 2018, A\&A, 616, A1

Gallazzi, A., Charlot, S., Brinchmann, J., White, S. D. M., \& Tremonti, C. A. 2005, MNRAS, 362, 41
Garrison-Kimmel, S., Bullock, J. S., Boylan-Kolchin, M., \& Bardwell, E. 2017a, MNRAS, 464, 3108

Garrison-Kimmel, S., Hopkins, P. F., Wetzel, A., et al. 2018, MNRAS, 481,4133

Garrison-Kimmel, S., Hopkins, P. F., Wetzel, A., et al. 2019, MNRAS, 487, 1380

Garrison-Kimmel, S., Wetzel, A., Bullock, J. S., et al. 2017b, MNRAS, 471,1709

Gómez, F. A., Helmi, A., Brown, A. G. A., \& Li, Y.-S. 2010, MNRAS, 408, 935

Grillmair, C. J., \& Carlin, J. L. 2016, in Tidal Streams in the Local Group and Beyond, ed. H. J. Newberg \& J. L. Carlin (Berlin: Springer), 87

Guedes, J., Callegari, S., Madau, P., \& Mayer, L. 2011, ApJ, 742, 76

Helmi, A., Babusiaux, C., Koppelman, H. H., et al. 2018, Natur, 563, 85

Helmi, A., \& White, S. D. M. 1999, MNRAS, 307, 495

Helmi, A., White, S. D. M., de Zeeuw, P. T., \& Zhao, H. 1999, Natur, 402, 53

Helmi, A., White, S. D. M., \& Springel, V. 2003, MNRAS, 339, 834

Herzog-Arbeitman, J., Lisanti, M., Madau, P., \& Necib, L. 2018a, PhRvL, 120, 041102

Herzog-Arbeitman, J., Lisanti, M., \& Necib, L. 2018b, JCAP, 1804, 052

Hopkins, P. F. 2015, MNRAS, 450, 53

Hopkins, P. F., Narayanan, D., \& Murray, N. 2013, MNRAS, 432, 2647

Hopkins, P. F., Wetzel, A., Kereš, D., et al. 2018, MNRAS, 480, 800

Ivezić, Z., Goldston, J., Finlator, K., et al. 2000, AJ, 120, 963

Johnston, K. V., Hernquist, L., \& Bolte, M. 1996, ApJ, 465, 278

Johnston, K. V., Spergel, D. N., \& Hernquist, L. 1995, ApJ, 451, 598

Katz, N., \& White, S. D. M. 1993, ApJ, 412, 455

Kirby, E. N., Cohen, J. G., Guhathakurta, P., et al. 2013, ApJ, 779, 102

Klypin, A. A., Trujillo-Gomez, S., \& Primack, J. 2011, ApJ, 740, 102

Kroupa, P. 2001, MNRAS, 322, 231

Krumholz, M. R., \& Gnedin, N. Y. 2011, ApJ, 729, 36

Kuhlen, M., Lisanti, M., \& Spergel, D. N. 2012, PhRvD, 86, 063505

Kuhlen, M., Weiner, N., Diemand, J., et al. 2010, JCAP, 1002, 030

Leitherer, C., Ekström, S., Meynet, G., et al. 2014, ApJS, 212, 14

Leitherer, C., Schaerer, D., Goldader, J. D., et al. 1999, ApJS, 123, 3

Lindegren, L., Lammers, U., Bastian, U., et al. 2016, A\&A, 595, A4

Lisanti, M., \& Spergel, D. N. 2012, PDU, 1, 155

Lisanti, M., Spergel, D. N., \& Madau, P. 2015, ApJ, 807, 14

Ma, X., Hopkins, P. F., Faucher-Giguere, C.-A., et al. 2016, MNRAS, 456,2140

Ma, X., Hopkins, P. F., Wetzel, A. R., et al. 2017, MNRAS, 467, 2430

Maciejewski, M., Vogelsberger, M., White, S. D. M., \& Springel, V. 2011, MNRAS, 415, 2475

McCarthy, I. G., Font, A. S., Crain, R. A., et al. 2012, MNRAS, 420, 2245

McConnachie, A. W. 2012, AJ, 144, 4

Myeong, G. C., Evans, N. W., Belokurov, V., Amorisco, N. C., \& Koposov, S. 2018a, MNRAS, 475, 1537

Myeong, G. C., Evans, N. W., Belokurov, V., Sanders, J. L., \& Koposov, S. E. 2018b, arXiv: 1805.00453

Myeong, G. C., Evans, N. W., Belokurov, V., Sanders, J. L., \& Koposov, S. E. 2018c, ApJL, 863, L28 
Necib, L., Lisanti, M., \& Belokurov, V. 2019, ApJ, 874, 3

O'Hare, C. A. J., McCabe, C., Evans, N. W., Myeong, G., \& Belokurov, V. 2018, PhRvD, 98, 103006

Onorbe, J., Garrison-Kimmel, S., Maller, A. H., et al. 2014, MNRAS, 437, 1894

Peñarrubia, J., Navarro, J. F., \& McConnachie, A. W. 2008, AN, 329, 934

Pérez, F., \& Granger, B. E. 2007, CSE, 9, 21

Pillepich, A., Madau, P., \& Mayer, L. 2015, ApJ, 799, 184

Purcell, C. W., Bullock, J. S., \& Zentner, A. R. 2007, ApJ, 666, 20

Purcell, C. W., Zentner, A. R., \& Wang, M.-Y. 2012, JCAP, 8, 027

Robertson, B., Bullock, J. S., Font, A. S., Johnston, K. V., \& Hernquist, L. 2005, ApJ, 632, 872

Sanderson, R. E., Garrison-Kimmel, S., Wetzel, A., et al. 2018a, ApJ, 869, 12
Sanderson, R. E., Wetzel, A., Loebman, S., et al. 2018b, arXiv:1806.10564 Springel, V. 2005, MNRAS, 364, 1105

Springel, V., Wang, J., Vogelsberger, M., et al. 2008, MNRAS, 391, 1685 Su, K.-Y., Hopkins, P. F., Hayward, C. C., et al. 2017, MNRAS, 471, 144 Vogelsberger, M., Helmi, A., Springel, V., et al. 2009, MNRAS, 395, 797 Vogelsberger, M., \& White, S. D. M. 2011, MNRAS, 413, 1419

Wang, J., Navarro, J. F., Frenk, C. S., et al. 2011, MNRAS, 413, 1373

Wetzel, A. R., Hopkins, P. F., Kim, J.-h., et al. 2016, ApJL, 827, L23

White, S. D. M., \& Rees, M. J. 1978, MNRAS, 183, 341

Yanny, B., Newberg, H. J., Kent, S., et al. 2000, ApJ, 540, 825

Zemp, M., Diemand, J., Kuhlen, M., et al. 2009, MNRAS, 394, 641

Zolotov, A., Willman, B., Brooks, A. M., et al. 2009, ApJ, 702, 1058 